\title{
Recruitment of inflammatory monocytes by senescent fibroblasts inhibits antigen-specific tissue immunity during human aging
}

\author{
Emma S. Chambers ${ }^{1,5,6}$, Milica Vukmanovic-Stejic ${ }^{1,6}$, Barbara B. Shih², Hugh Trahair (1) \\ Priya Subramanian', Oliver P. Devine', James Glanville ${ }^{3}{ }^{3}$, Derek Gilroy ${ }^{3}$, Malcolm H. A. Rustin ${ }^{4}$, \\ Tom C. Freeman ${ }^{2}$, Neil A. Mabbott $\mathbb{D}^{2}$ and Arne N. Akbar ${ }^{1}{ }^{\square}$
}

\begin{abstract}
We have previously shown that healthy older adults exhibit reduced cutaneous immune responses during a varicella zoster virus (VZV) antigen challenge that correlated with a nonspecific inflammatory response to the injection itself. Here we found that needle damage during intradermal injections in older adults led to an increase in the number of cutaneous senescent fibroblasts expressing CCL2, resulting in the local recruitment of inflammatory monocytes. These infiltrating monocytes secreted prostaglandin E2, which inhibited resident memory $\mathrm{T}$ cell activation and proliferation. Pretreatment of older participants with a p38 mitogen-activated protein kinase inhibitor in vivo decreased CCL2 expression and inhibited monocyte recruitment and secretion of prostaglandin E2. This coincided with an increased response to VZV antigen challenge in the skin. Our results point to a series of molecular and cellular mechanisms that link cellular senescence, tissue damage, excessive inflammation and reduced immune responsiveness in human skin and demonstrate that tissue-specific immunity can be restored in older adults by short-term inhibition of inflammatory responses.
\end{abstract}

mmunity decreases during aging as demonstrated by the increased susceptibility to infections including pneumonia and influenza, reactivation of latent infections such as varicella zoster virus (VZV), decreased vaccine efficacy and increased incidence of cancer ${ }^{1-3}$. In addition, there is an increase in low-grade systemic inflammation in older humans, termed inflammaging, characterized by high serum levels of the inflammatory cytokines interleukin (IL)-6, IL-1 $\beta$, tumor-necrosis factor- $\alpha$ and C-reactive protein $(\mathrm{CRP})^{4}$. This elevated expression of inflammatory proteins is a strong predictor for frailty and mortality ${ }^{5,6}$. Mechanisms that contribute to inflammaging are multifactorial and include increased numbers of senescent cells, which secrete many inflammatory proteins as part of the senescence-associated secretory phenotype (SASP), increased gut permeability and subsequent lipopolysaccharide (LPS; a Toll-like receptor 4 ligand) exposure, expression of damage-associated molecular pattern molecules and failure of immune resolution 7 .

Cutaneous challenge with recall antigen is often used as a measure of effective immunity. Older people have reduced responses to challenge with various recall antigens including tuberculin PPD, Candida albicans and $\mathrm{VZV}^{8-11}$. We showed previously that this decrease was a result of heightened inflammatory responses in the skin of older people during immune challenge and not from changes in antigen-specific circulating or skin-resident memory $\mathrm{T}$ cells $\left(\mathrm{T}_{\mathrm{RM}}\right)^{12}$. However, the mechanism by which inflammation inhibits antigen-specific immunity in the skin is unknown.

Here we show that when older adults are challenged in the skin with VZV antigens, the tissue damage caused by the injection of the antigen itself induces senescent fibroblasts to recruit CCR $2^{+} \mathrm{CD} 14^{+}$ monocytes from the circulation. These infiltrating monocytes express cyclooxygenase 2 (COX2) and produce prostaglandin E2 $\left(\mathrm{PGE}_{2}\right)$ that inhibits activation and proliferation. Pretreatment of older adults with an oral p38 mitogen-activated protein kinase (MAPK) inhibitor inhibited monocyte recruitment and $\mathrm{PGE}_{2}$ production during the immune response to VZV. This resulted in a significantly enhanced VZV-specific mediated delayed-type hypersensitivity response associated with the activation and expansion of $\mathrm{T}$ cell numbers in the skin. These data identify a broad mechanism by which even mild tissue damage may induce inflammation in the tissues of older people that can inhibit immunity. An important consideration is whether the short-term blockade of inflammation would enhance the immune responses in older people at early stages of infection when the pathogen induces damage and excessive inflammation that may retard subsequent immune responsiveness.

\section{Results}

Inappropriate response to tissue damage in the skin during aging. Old ( $\geq 65$ years) and young ( $<40$ years) adults were recruited for this study (donor characteristics are available in Supplementary Table 1). Although more females were included in the older adult group, there was no difference in response between the different genders within this group (Extended Fig. 1a). Volunteers were injected intradermally with VZV skin test antigen in one arm (for the clinical score) and with saline $(0.9 \% \mathrm{NaCl})$ in the other arm as a control. Biopsies (5- $\mathrm{mm})$ were collected from the injection sites $6 \mathrm{~h}$ after injection and compared to biopsies of normal (unmanipulated) skin (Fig. 1a). The VZV injection site was assessed for clinical score $72 \mathrm{~h}$ after injection and the induration, palpability and

'Division of Infection and Immunity, University College London, London, UK. ${ }^{2}$ The Roslin Institute and Royal (Dick) School of Veterinary Studies, University of Edinburgh, Easter Bush, Midlothian, UK. ${ }^{3}$ Division of Medicine, University College London, London, UK. ${ }^{4}$ Department of Dermatology, Royal Free Hospital, London, UK. ${ }^{5}$ Present address: Centre for Immunobiology, Blizard Institute, Queen Mary University of London, London, UK. ${ }^{6}$ These authors

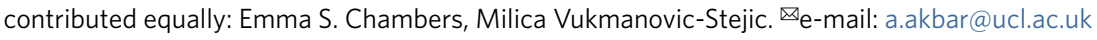



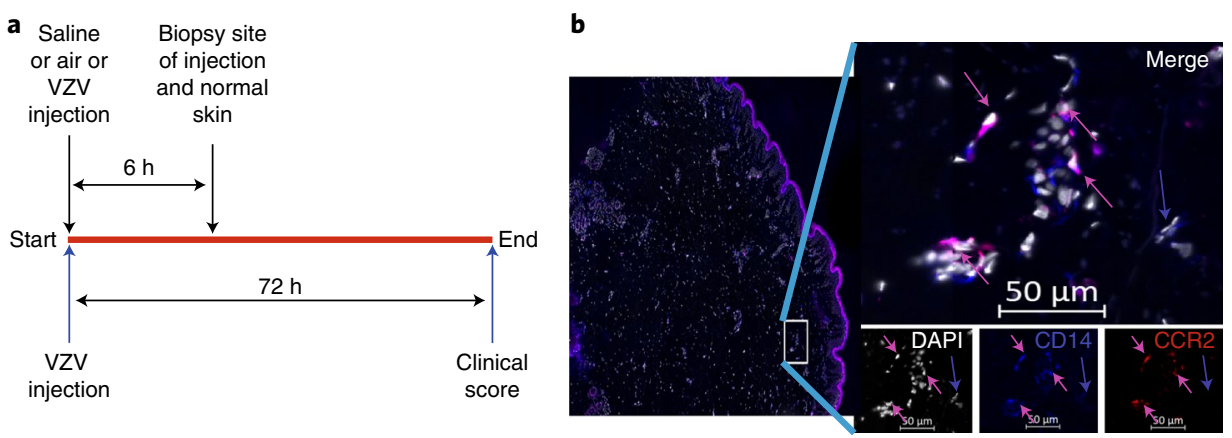

c
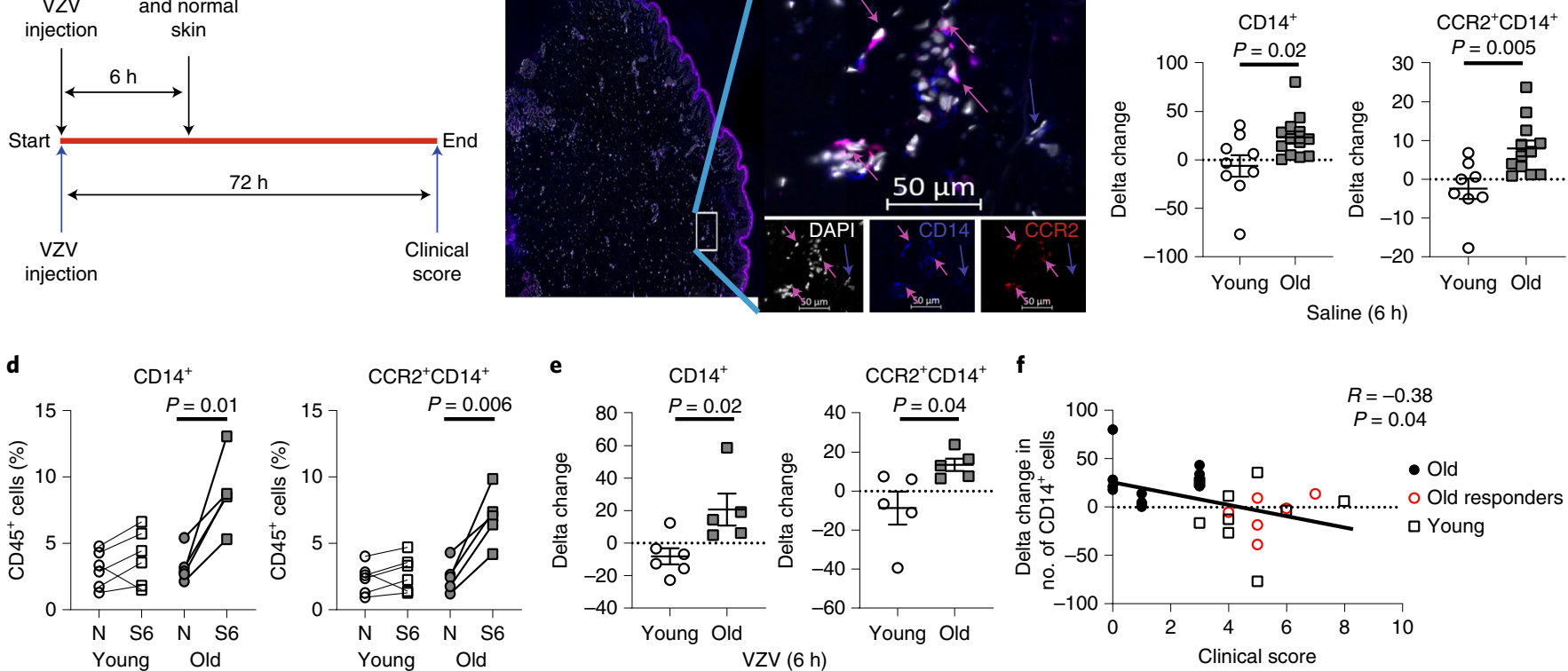

Fig. 1 | Recruitment of monocytes to injection sites in the skin. a, Schematic of visits for blood and skin sampling by old and young participants. b, Representative image of a skin section stained with DAPI (white), CCR2 (red) and CD14 (blue); pink arrows indicate CD14 and CCR2 co-staining, and the blue arrow indicates CD14 single staining. c, Cumulative delta change in the number of $\mathrm{CD} 14^{+}$or $\mathrm{CCR} 2^{+} \mathrm{CD} 14^{+}$cells in the skin after saline injection ( $n=9$ young and $n=13$ old biologically independent individuals). d, Cumulative data showing the frequency of monocytes in normal ( $N$ ) and saline $(6 \mathrm{~h} ; \mathrm{S6})$ skin biopsies collected from young (white; $n=6$ ) and old (gray; $n=5$ ) individuals as assessed by flow cytometry. Cells were identified as mononuclear phagocytes if they were CD45 lineage but negative for CD3, CD19, CD20, CD56 and HLA-DR ${ }^{+}$. The CD14+ and CCR2 ${ }^{+}$CD14 ${ }^{+}$cells were assessed as a percentage of $\mathrm{CD} 45^{+}$cells present in the skin. e, Cumulative delta change in the number of CD14+ ${ }^{+}$or CCR2 ${ }^{+} \mathrm{CD} 14^{+}$cells in VZV-injected skin ( $n=5$ young and $n=5$ old biologically independent individuals) as compared to normal skin. $\mathbf{f}$, Correlation between delta change in the number of $\mathrm{CD}_{14}{ }^{+}$cells in the skin after saline injection as compared to normal skin in relation to their VZV clinical response. Old donors are represented by black circles (VZV score: $0-3 ; n=13$ ), old responders by red circles (VZV clinical score: $\geq 4 ; n=6)$ and young donors $(n=9)$ by black squares. Data are the mean \pm s.e.m. and were analyzed using an unpaired $t$-test (c and $\mathbf{e}$ ) and Spearman's correlation (f).

change in erythema from baseline were measured and scored, as described before ${ }^{11}$. We showed previously that mononuclear phagocyte numbers were increased at the site of saline injection in old but not young participants, and that this accumulation negatively correlated with the magnitude of antigen-specific responses induced in the contralateral arm of the same individual ${ }^{9}$. Multiparametric flow cytometry on disaggregated skin biopsies from older donors showed no change in the number of $\mathrm{CD} 11 \mathrm{c}^{+}, \mathrm{CD} 1 \mathrm{c}^{+}$or $\mathrm{BDCA}-3^{+}$ dendritic cells (DCs) in response to saline challenge (Extended Data Fig. 2a-d). Neutrophils, as determined by CD66b expression by immunofluorescence, or CD $45^{+}$lineage ${ }^{-} \mathrm{CD} 16^{+} \mathrm{HLA}-\mathrm{DR} \mathrm{R}^{-}$cells, as measured by flow cytometry, were observed at very low numbers in normal skin, which did not significantly change after saline injection (Extended Data Figs. 3a,b and 4a,b). These data imply that the increase in mononuclear phagocytes was due to accumulation of monocytes.

To enumerate the changes in monocyte numbers following saline injection, we assessed paired samples and expressed data as the delta change from normal skin. This was to mitigate against interpersonal variation in the magnitude of responses observed (Extended Data Fig. 5a). CD14 was used to identify monocytes in these experiments as it is expressed by the majority of peripheral blood monocytes (classical and intermediate), while $\mathrm{CD} 16^{+} \mathrm{CD} 14^{-}$monocytes were relatively rare in the skin (Extended Data Fig. 4a). There was a significant increase in $\mathrm{CD} 14^{+}$cells in saline-injected skin at $6 \mathrm{~h}$ after injection (Fig. 1b,c). We next investigated the expression of CCR2, a chemokine receptor for CCL2, which is highly expressed by $\mathrm{CD} 14^{+}$monocytes $^{13,14}$. There was a significant increase in the number of $\mathrm{CD} 14^{+} \mathrm{CCR} 2^{+}$cells in saline-injected skin as compared to normal skin in old but not young participants (Fig. 1c; larger images are available in Supplementary Fig. 1a,b). This response to saline was transient, as $24 \mathrm{~h}$ after injection, similar frequencies of monocytes were detected in normal and saline-injected skin (Extended Data Fig. 5b). Multiparametric flow cytometry analysis of normal and saline biopsies, using the gating strategy shown in Extended Data Fig. 4a, confirmed a significant increase in $\mathrm{CD} 14^{+}$ and $\mathrm{CCR} 2^{+} \mathrm{CD} 14^{+}$cells in saline-injected skin of older adults (Fig. 1d). We also observed a smaller population of HLA-DR ${ }^{+}$cells that was $\mathrm{CCR} 2^{+} \mathrm{CD} 14^{-}$in normal skin; however, this population did not significantly change after saline injection (Extended Data Fig. 4c). Further phenotyping of the HLA-DR ${ }^{+}$population with CD86, CLA, SLAN, CD16 and $\mathrm{CX}_{3} \mathrm{CR} 1$ showed that their expression was similar before and after saline injections. The only populations that changed in the older participants after saline injection were those that expressed CD14 and CCR2, suggesting that it was an increase in the number of $\mathrm{CD} 14^{+} \mathrm{CCR} 2^{+}$monocytes rather than a change in their phenotype (Extended Data Fig. 4d-f).

To investigate whether this inflammatory response after saline injection in older adults was due to injection with sodium chloride itself or a response to needle injury, we performed intradermal injections with the same volume of air instead of saline $(20 \mu \mathrm{l})$. Air injection also induced a significant increase in the number of monocytes at the site of challenge in old but not young adults compared to normal skin (Extended Data Fig. 5c). The accumulation of monocytes was also observed $6 \mathrm{~h}$ later at the site of VZV injection in old but not young adults (Fig. 1e). Taken together, these data suggest 
a
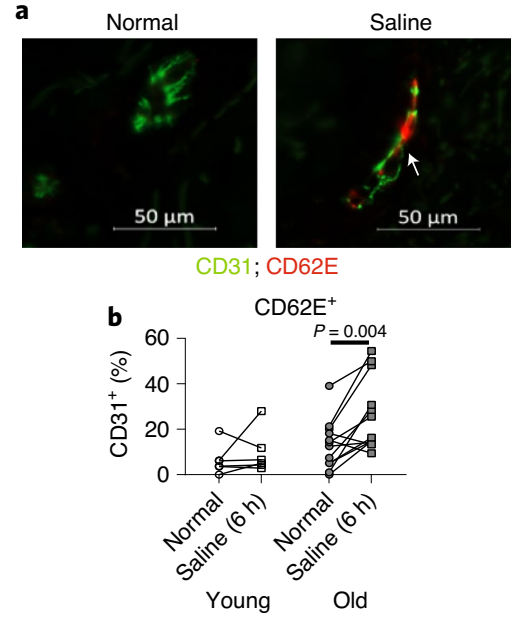

c

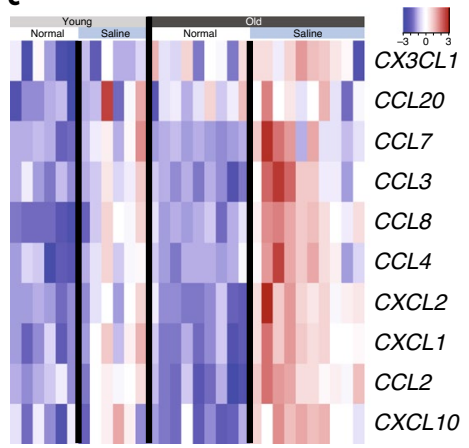

d

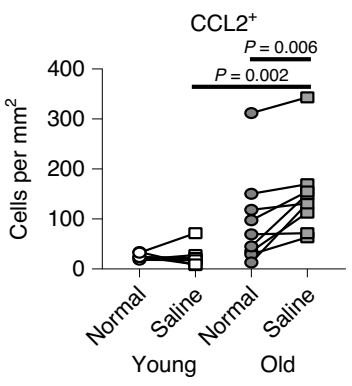

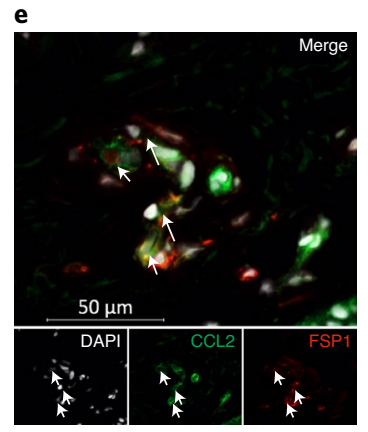

f

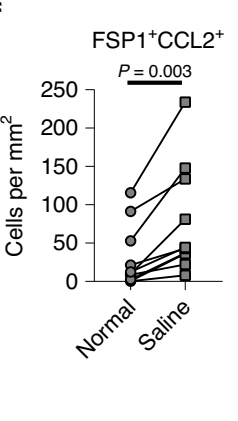

g

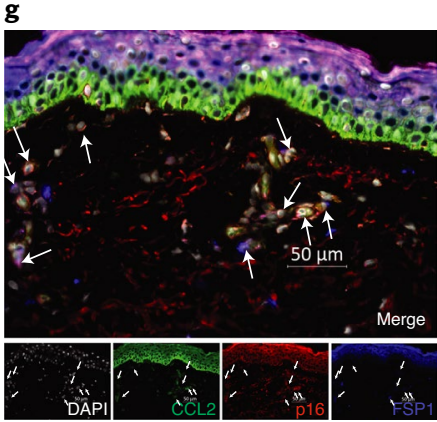

h

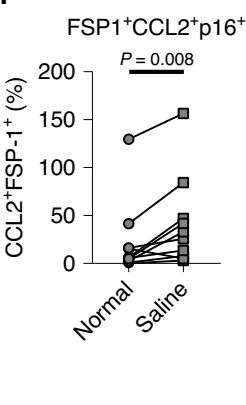

i

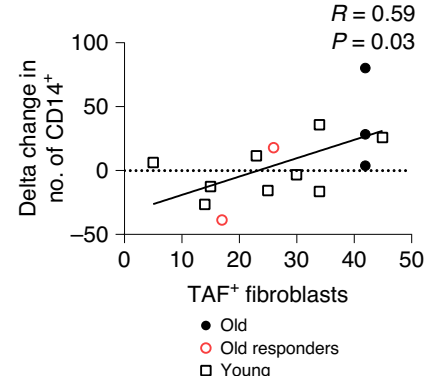

Fig. 2 | Monocyte chemoattractant expression after saline injection in the skin. a, Image is representative of normal and saline-injected skin stained with CD31 (green) and CD62E (red). b, Cumulative data showing the frequency of CD31+ endothelial cells expressing CD62E in young (white; $n=6$ ) and old (gray; $n=12$ ) donors. c, Heat map showing expression of monocyte chemokines in normal and saline-injected skin of young and older donors. d, Cumulative data showing the number of $\mathrm{CCL} 2^{+}$cells in young (white; $n=7$ ) and old (gray; $n=9$ ) donors. e, Representative image of a skin section stained with DAPI (white), CCL2 (green) and FSP1 (red); arrows indicate double-positive cells. f, Cumulative data showing the number of FSP1+CCL2+ cells in normal and saline-injected skin from older donors $(n=10)$. $\mathbf{g}$, Representative image of a skin section stained with DAPI (white), CCL2 (green), p16 (red) and FSP1 (blue); arrows indicate FSP1 ${ }^{+} \mathrm{CCL} 2^{+} \mathrm{p} 16^{+}$cells. $\mathbf{h}$, Cumulative data showing the number of FSP1 ${ }^{+} \mathrm{CCL}{ }^{+} \mathrm{p} 16+{ }^{+}$cells $(n=10)$. $\mathbf{i}$, Correlation between the number of senescent fibroblasts in normal skin (defined as being positive for TAF) and the change in CD14 ${ }^{+}$monocytes after saline injection in old donors (black circles; $n=3$ ), old responders (red circles; $n=2$ ) and young donors (black squares; $n=8$ ). Data were assessed using a paired $t$-test (b,d,f and $\mathbf{h}$ ) and Spearman's rank correlation (i).

that older participants have a heightened inflammatory response to mild skin injury that is not observed in young adults.

We previously showed that $>85 \%$ of older individuals have a low VZV response (clinical score: $0-3$ ), while $>85 \%$ of young individuals have a score of 3-10 (refs. ${ }^{8,12}$ ). However, a small proportion of older people $(\sim 15 \%)$ show good cutaneous VZV responsiveness (score: $>3$ ), a group we designated as 'old responders'. To assess whether the $\mathrm{CD} 14^{+}$monocyte infiltration was a characteristic of all aged individuals or only those with reduced VZV-specific immunity, we investigated the accumulation of these cells after saline in old VZV responders and low responders (Fig. 1f). An increase in monocytes at the site of challenge was only observed in older adults with a low clinical score, while those with a good clinical score behaved similarly to young individuals with low monocyte infiltration. Taken together, we observed a significant negative correlation between the fold change in $\mathrm{CD}_{1} 4^{+}$monocytes in response to saline challenge and the VZV clinical score $(R=-0.38$ and $P=0.04$; Fig. 1 e), and a similar correlation was observed between $\mathrm{CD} 14^{+} \mathrm{CCR} 2^{+}$ cells and clinical score (Supplementary Fig. 2). Collectively, this indicates that early recruitment of monocytes to the site of skin challenge in older adults was associated with decreased antigenspecific immunity.
Senescent stromal cells contribute to recruitment of monocytes in older adults. We hypothesized that the increase in $\mathrm{CD} 14^{+}$cells in the skin was due to recruitment of circulating monocytes, since $\mathrm{CCR}^{+}$expression supported the possibility of recruitment in response to CCL2. The alternative explanation that this was a consequence of the proliferation of local tissue-resident macrophages (identified as $\mathrm{CD}_{163}{ }^{+}$cells), however, was not observed (Extended Data Fig. $6 \mathrm{a}-\mathrm{c}$ ). This is in line with murine studies showing that dermal macrophages are constantly replenished from the monocyte pool rather than by proliferation ${ }^{15}$. We first investigated the activation status of the dermal endothelium to determine whether it was permissive for monocyte recruitment. CD62E, also known as E-selectin, was used as a marker of activated endothelium because it binds to sialylated carbohydrates expressed on leukocytes and facilitates their extravasation into the tissue. Saline-injected skin of old but not young adults exhibited increased CD62E expression on the $\mathrm{CD} 1^{+}$endothelium when compared with normal skin (Fig. 2a,b). Furthermore, transcriptional analysis of skin biopsies indicated increased expression of genes encoding monocyte chemoattractants, including CCL2 (encodes one of the main monocyte chemoattractants), CXCL10, CXCL2 and CXCL1 in old but not young adults in response to saline injection (Fig. 2c). Increased CCL2 expression 
a

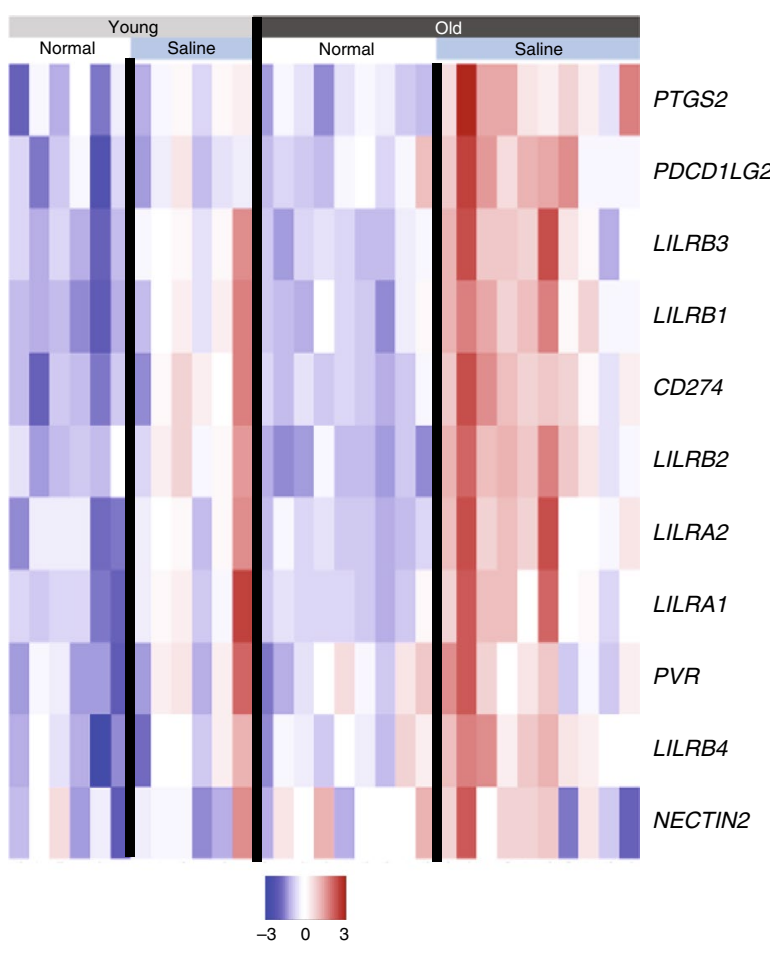

b

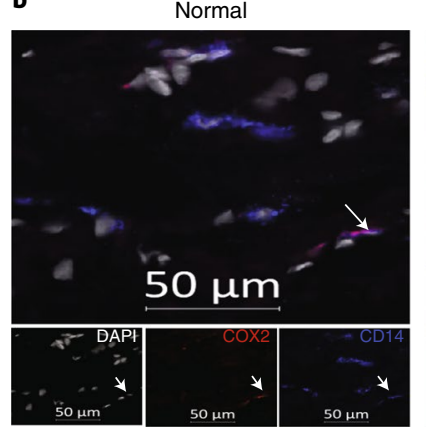

C

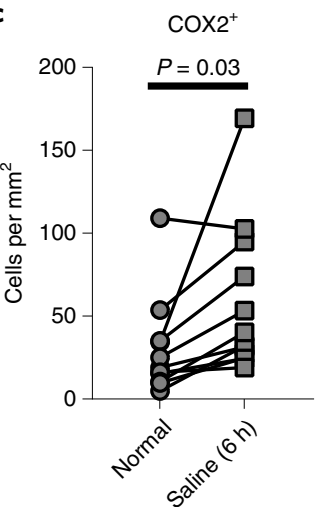

Saline

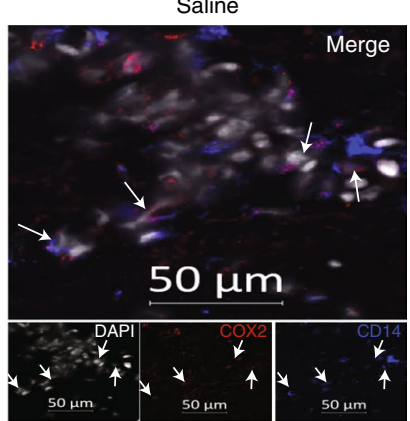

d

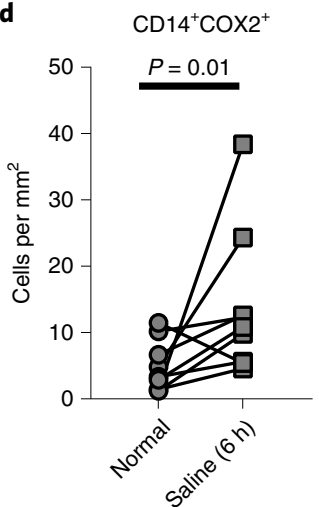

e

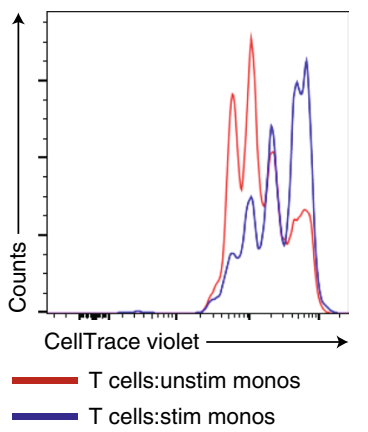

h

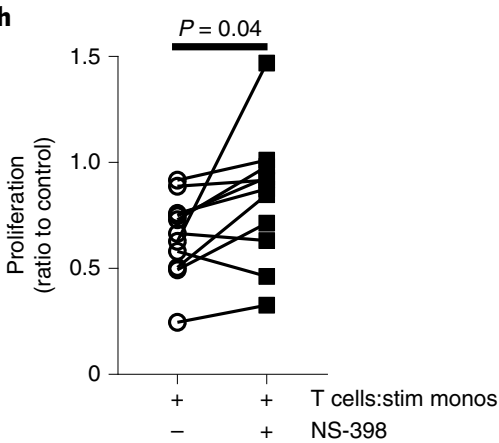

f

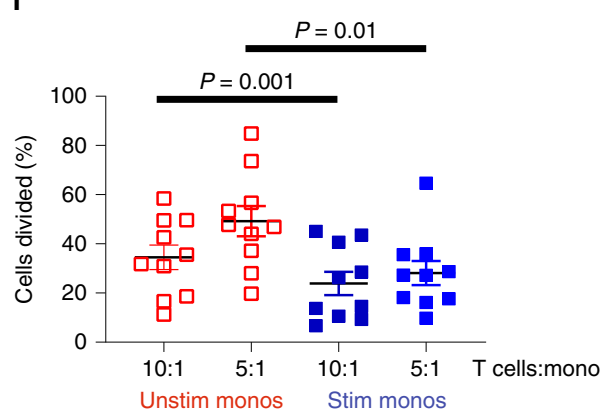

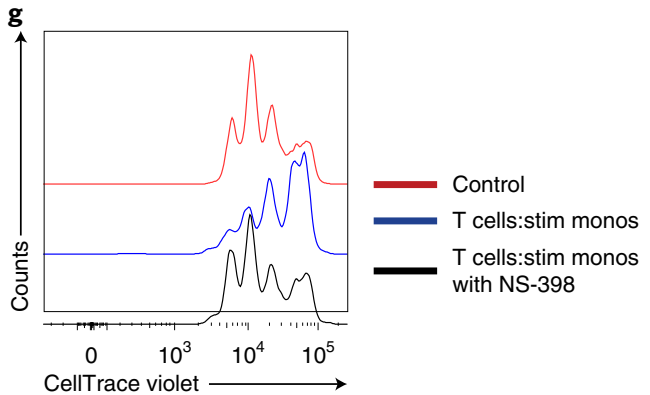
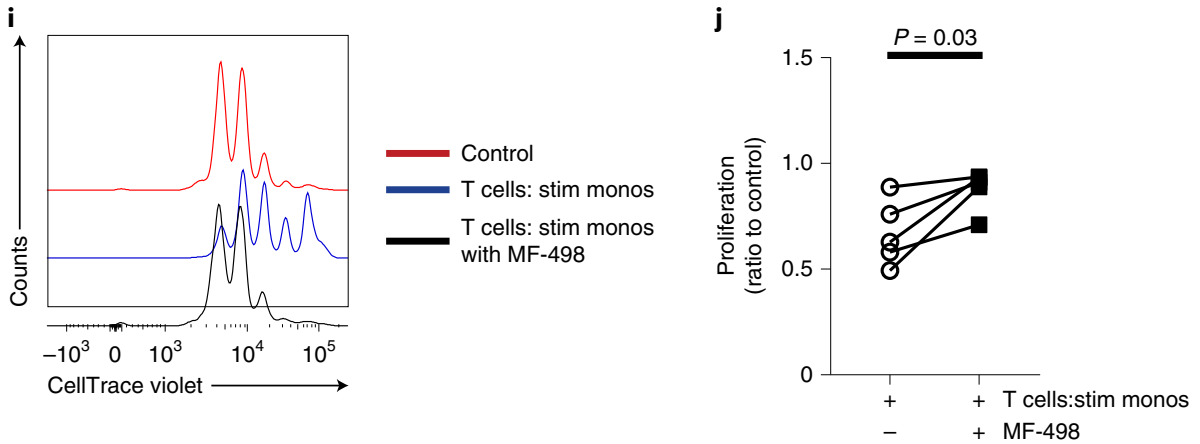

Fig. 3 | CD14 ${ }^{+} \mathrm{COX2}^{+}$monocytes inhibit T cell proliferation via PGE ${ }_{2}$ a, Heat map showing the expression of genes associated with inhibitory mechanisms in normal and saline-injected skin of old and young donors. b, Representative image stained with DAPI (white), COX2 (red) and CD14 (blue) in normal and saline-injected skin. c, Cumulative data of total COX2-expressing cells $(n=11)$. d, The number of CD14+COX2+ cells in normal and saline-injected skin $(n=9)$. Monocytes were negatively isolated from the peripheral blood and cultured with and without LPS for $3 \mathrm{~h}$, then subsequently co-cultured with autologous T cells (pre-labeled with CellTrace Violet) that were activated with plate-bound CD3 and IL-2. Proliferation was assessed at day 4. e, Representative flow cytometry plot of CellTrace violet dilution in $\mathrm{CD} 4^{+} \mathrm{T}$ cells co-cultured with unstimulated and stimulated monocytes. $\mathbf{f}$, Cumulative data showing the percentage of cells divided $(n=10) \cdot \mathbf{g}-\mathbf{j}$, A similar co-culture experiment was performed in the presence or absence of the COX2 inhibitor NS-398 ( $n=11 ; \mathbf{g}$ and $\mathbf{h})$ or the EP4 receptor inhibitor MF-498 $(n=5 ; \mathbf{i}$ and $\mathbf{j})$. Controls were unstimulated monocytes co-cultured with T cells in the presence of drug. Each data point represents a biologically independent experiment. Data are the mean \pm s.e.m. in $\mathbf{f}$, analyzed using a paired $t$-test in $\mathbf{c}, \mathbf{d}, \mathbf{f}, \mathbf{h}$ and $\mathbf{j}$. 

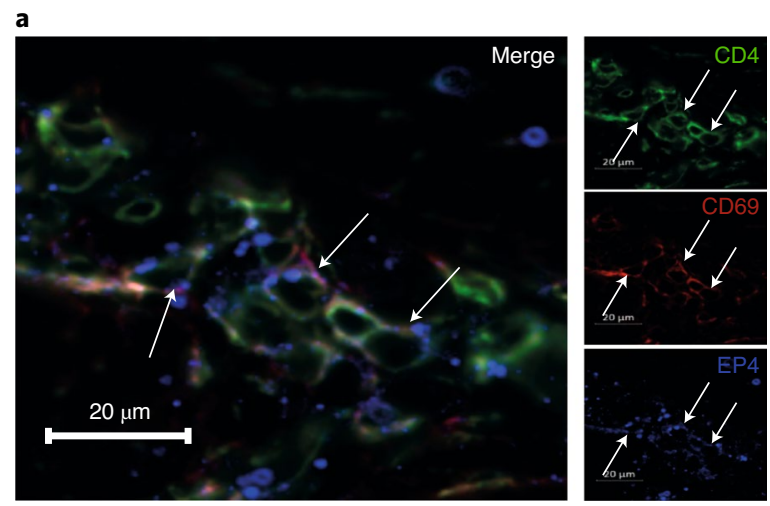

b
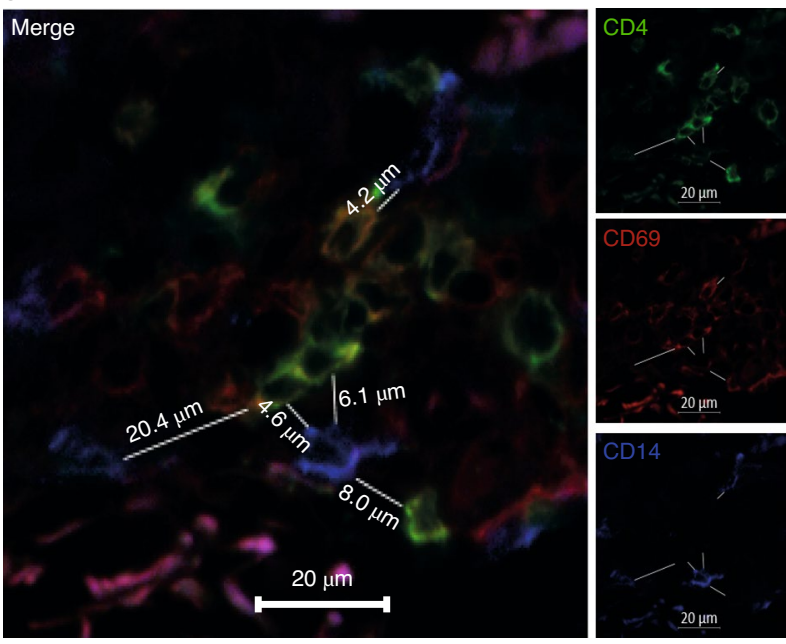

d

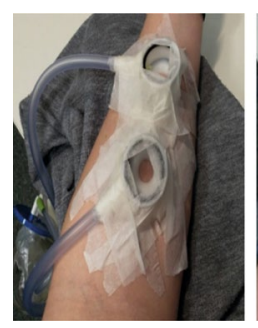

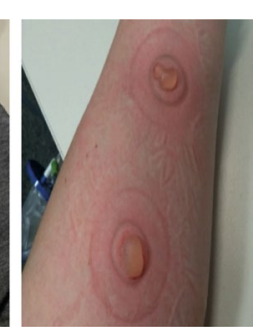

e

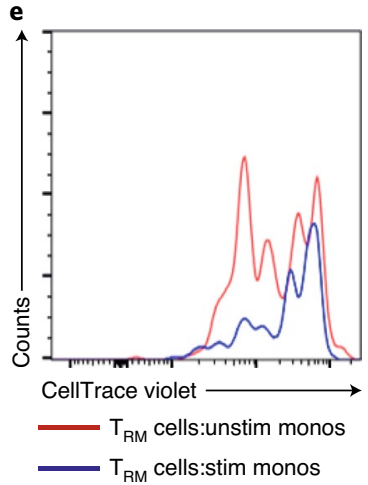

c

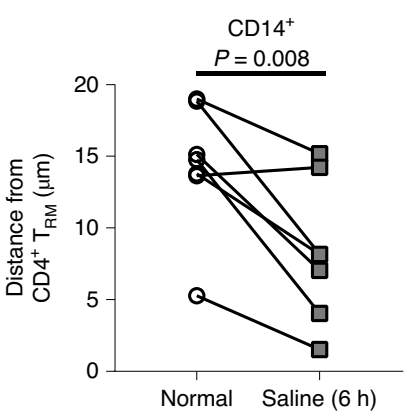

f

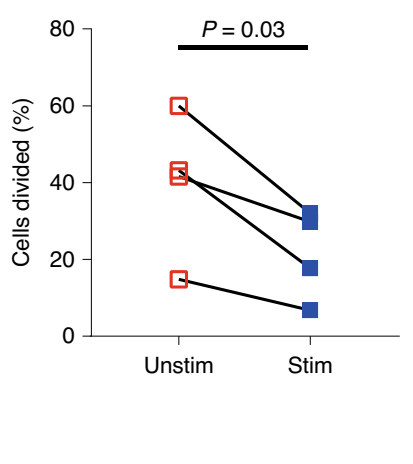

Fig. 4 | PGE 2 production by monocytes inhibits $\mathrm{CD}^{+} \mathbf{T}_{\mathrm{RM}}$ cell proliferation in skin. a, Representative staining of CD4 (green), CD69 (red) and EP4 (blue) in normal skin from eight independent donors; white arrows indicate CD4 ${ }^{+} T_{R M}$ cells that coexpressed EP4. $\mathbf{b}$, Representative staining showing colocalization of $C D 4^{+} T_{R M}$ cells and $\mathrm{CD}_{14}{ }^{+}$monocytes (blue). c,d, Cumulative data showing the distance of $C D 14^{+}$monocytes from $T_{R M}$ cells in normal and saline-injected skin from older adults $(n=7 ; \mathbf{c})$. Monocytes were negatively isolated from the peripheral blood and cultured with and without LPS for $3 \mathrm{~h}$ and then subsequently co-cultured with skin-resident $\mathrm{T}_{\mathrm{RM}}$ cells (pre-labeled with CellTrace Violet), which were collected from suction blisters (representative image in d) and then activated with plate-bound CD3 and IL-2. Proliferation was assessed at day 4. e, Representative flow cytometry plot of CellTrace violet dilution in $T_{R M}$ cells co-cultured with unstimulated (red line) or stimulated (blue line) monocytes. f, Cumulative data showing $\mathrm{CD}^{+} \mathrm{T}_{\mathrm{RM}}$ cell proliferation in the presence of stimulated or unstimulated monocytes, assessed at day $4(n=4)$. Data in $\mathbf{c}$ and $\mathbf{f}$ were analyzed with a paired $t$-test.

in saline-injected skin of older adults was confirmed by immunofluorescence staining of skin sections (Fig. 2d). As expected, there was no significant change in the expression of CCL2 cells in the skin of young adults after saline injection (Fig. 2d).

We next examined the source of monocyte chemoattractants and found that resident mononuclear phagocytes were not the main source of CCL2 (Extended Data Fig. 7a,b). Using fibroblast-specific protein (FSP) 1 as a marker of fibroblasts, we found a significant increase in the number of fibroblasts expressing CCL2 in the skin of older participants after saline injection as compared to normal uninjected skin (Fig. 2e,f; larger images are available in Extended Data Fig. 7c). It is known that senescent fibroblasts can produce 
CCL2 (ref. ${ }^{16}$ ). We and others have shown that there are increased numbers of senescent fibroblasts in the skin of older people ${ }^{16-18}$, and CCL2 is a known component of SASP ${ }^{19}$. Indeed, using p16 as a marker of senescence, we found that $\mathrm{FSP} 1^{+} \mathrm{p} 16^{+}$fibroblasts were the main source of CCL2 after saline injection of older donors (Fig. 2g,h and Extended Data Fig. 7d). Senescent fibroblasts made up $54.1 \%$ of $\mathrm{CCL} 2^{+} \mathrm{FSP} 1^{+}$cells in normal skin, increasing to $66.8 \%$ in saline-injected skin (Extended Data Fig. 7e). Although some non-senescent fibroblasts evidently expressed CCL2, our previous data showed that senescent fibroblasts produce significantly more CCL2 than the non-senescent fibroblasts ${ }^{16}$. Therefore, early production of SASP-related mediators by senescent fibroblasts after saline injection (including CCL2) may lead to the recruitment of inflammatory monocytes to the site of skin challenge. This interaction between senescent cells and monocyte recruitment is supported indirectly by the significant correlation between the number of senescent fibroblasts present in the skin, as determined by the number of FSP1 $1^{+}$cells positive for telomere-associated DNA damage foci (TAF; representative staining is available in Extended Data Fig. 8), and the extent of $\mathrm{CD} 14^{+}$monocyte infiltration after saline injection (Fig. 2i).

Monocytes inhibit $T_{\mathrm{RM}}$ cell proliferation through production of prostaglandin E2. We next investigated the mechanisms by which the recruitment of monocytes reduced antigen-specific immunity in older participants. We first assessed inhibitory pathways in normal and saline-injected skin using RNA sequencing (RNA-seq). We found that saline injection in older adults increased the expression of a number genes that encode immunoglobulin-like transcript (ILT) receptors such as LILRB3 (ILT5), LILRB2 (ILT4), LILRA2 (ILT1), LILRB4 (ILT3) and LILRB1 (ILT2); inhibitory ligands such as CD274 (programmed death ligand (PDL)-1 and PDCD1LG2 (PDL-2), ligand for the T cell receptor TIGIT and $P V R$, as well as inducible COX2 enzyme (PTGS2; Fig. 3a).

PTGS2 (encoding COX2) was one of the most highly upregulated genes in saline-injected skin of older participants ${ }^{9}$, and elevated COX2 expression following saline injection was confirmed at the protein level by immunohistology (Fig. 3b,c and Extended Data Fig. 9a). When staining for COX2 was performed in combination with $\mathrm{CD} 14$, there was a significant increase in $\mathrm{CD} 14^{+} \mathrm{COX} 2^{+}$cells in saline-injected as compared to normal skin (Fig. $3 \mathrm{~d}$ ). No significant difference in the number of $\mathrm{CD} 14^{-} \mathrm{COX}^{+}$cells was seen (Extended Data Fig. 9b), indicating that this increase in COX2 was specific to CD14 ${ }^{+}$monocytes.

To demonstrate that COX2-expressing monocytes can inhibit the activation of $\mathrm{CD}^{+} \mathrm{T}$ cells, we first induced COX2 expression in monocytes isolated from peripheral blood by stimulation with LPS $\left(1 \mathrm{ng} \mathrm{m}^{-1}\right)$ for $3 \mathrm{~h}$. LPS treatment significantly increased COX2 expression as compared to untreated controls (Supplementary Fig. 3 ${ }^{20,21}$. Unstimulated and LPS-stimulated monocytes were then co-cultured with autologous peripheral $\mathrm{CD}^{+} \mathrm{T}$ cells in the presence of anti-CD3 and IL-2, and proliferation was assessed after $4 \mathrm{~d}$. Stimulated monocytes inhibited $\mathrm{CD}^{+} \mathrm{T}$ cell proliferation as compared to control (unstimulated) monocytes (Fig. 3e,f). However, production of the cytokines interferon- $\gamma$, IL- 2 and IL-10 was not affected at day 4 after culture. The COX2-specific inhibitor NS-398 was then used to determine if COX2 expression was involved in the inhibition of $\mathrm{CD}^{+} \mathrm{T}$ cell proliferation. Treatment with NS-398 led to a significant increase in the proliferation of the $\mathrm{CD} 4^{+} \mathrm{T}$ cells (Fig. 3g,h and Supplementary Fig. 4a). COX2 is involved in the production of a range of lipid mediators, the most common being $\mathrm{PGE}_{2}$ (ref. ${ }^{22}$ ). To establish if $\mathrm{PGE}_{2}$ was involved in the inhibition of $\mathrm{CD}^{+}$ T cell proliferation, we blocked the receptor for $\mathrm{PGE}_{2}$ (EP4) on $\mathrm{CD}_{4}^{+}$ $\mathrm{T}$ cells using the inhibitor MF-498. Blockade of $\mathrm{PGE}_{2}$ signaling also significantly increased the proliferation of the $\mathrm{CD} 4^{+} \mathrm{T}$ cells in the presence of inflammatory monocytes (Fig. 3h,i and Supplementary Fig. 4b). To confirm that $\mathrm{PGE}_{2}$ did not alter the monocyte phenotype, we cultured monocytes with $\mathrm{PGE}_{2}$ and assessed expression of their inhibitory receptors (CD112, CD155, galectin 9, ILT3, PDL-1 and PDL-2) and production of cytokines. We did not observe any significant effects of $\mathrm{PGE}_{2}$ treatment on the expression of these receptors in monocytes, confirming that $\mathrm{PGE}_{2}$ directly affected $\mathrm{CD}^{+} \mathrm{T}$ cells. To assess if the above observations were relevant in the skin of older donors in vivo, we investigated EP4 expression by skin-resident $\mathrm{CD} 4^{+} \mathrm{CD} 69^{+} \mathrm{T}_{\mathrm{RM}}$ cells in older volunteers (Fig. 4a). The majority of CD4 ${ }^{+} \mathrm{T}_{\mathrm{RM}}$ cells expressed EP4 (81.2\%), indicating that they have the capacity to respond to $\mathrm{PGE}_{2}$ produced by monocytes. We next assessed the colocalization of $\mathrm{CD} 14^{+}$monocytes and $\mathrm{CD}^{+} \mathrm{T}_{\mathrm{RM}}$ cells in normal and saline-injected skin from older participants. In normal skin, $\mathrm{CD} 14^{+}$monocytes were, on average, $15.86-\mu \mathrm{m}$ away from $\mathrm{CD} 4^{+} \mathrm{T}_{\mathrm{RM}}$ cells; however, after saline injection, the monocytes were significantly closer to the $\mathrm{CD} 4^{+} \mathrm{T}_{\mathrm{RM}}$ cells $(9.47 \mu \mathrm{m}$; Fig. $4 \mathrm{~b}, \mathrm{c})$. This increased proximity was likely due to an increase in the number of monocytes in the skin after saline injection. To confirm that monocytes have the ability to suppress skin $\mathrm{CD}^{+}{ }^{+} \mathrm{T}_{\mathrm{RM}}$ cell proliferation, $\mathrm{CD} 4^{+} \mathrm{T}_{\mathrm{RM}}$ cells were isolated from the skin using suction blister technology, as described previously (Fig. $4 d)^{11}$. $\mathrm{CD}^{+} \mathrm{T}_{\mathrm{RM}}$ cells in skin were then pre-labeled with CellTrace Violet and stimulated with CD3 and IL-2 in the presence of either stimulated or unstimulated monocytes for $4 \mathrm{~d}$. We observed a significant reduction in $\mathrm{CD}^{+} \mathrm{T}_{\mathrm{RM}}$ cell proliferation in the presence of stimulated as compared to unstimulated monocytes (Fig. 4e,f), supporting the hypothesis that $\mathrm{COX} 2^{+}$monocytes can suppress $\mathrm{T}_{\mathrm{RM}}$ cell proliferation.

p38 MAPK inhibition significantly reduces CCL2 production from senescent fibroblasts. We showed previously that treatment of older participants with the anti-inflammatory p38 MAPK inhibitor losmapimod significantly enhanced the response to VZV antigens in the skin 9 . To investigate if p38 MAPK inhibition altered CCL2 production and inflammatory monocyte recruitment in the skin, RNA-seq analysis was performed on biopsies collected from normal skin and from skin $6 \mathrm{~h}$ after saline injection, both before and after losmapimod treatment (see Fig. 5a for study design). We stratified participants in the RNA-seq analysis according to the extent of clinical score improvement (Fig. 5b). A list of all differentially expressed genes can be found in Supplementary Table 4. Saline injection resulted in a large pro-inflammatory response with a significant increase in the expression of inflammatory associated genes such as CCL2, CAFCL8, FOSL1, IL1B and TREM1 at $6 \mathrm{~h}$ before

Fig. 5 | Reduced inflammatory monocyte recruitment to the skin by pretreatment with losmapimod. a, Losmapimod clinical study diagram. Black arrows indicate study visits, red arrows indicate blood samples and blue arrows indicate skin biopsies. $\mathbf{b}$, Color coding of the clinical response of each individual after VZV challenge before and after losmapimod pretreatment (pink/brown); no improvement in clinical score is represented by white, while the biggest improvement in clinical score is shown in dark green. c, This coding was used in conjunction with the transcriptomic signatures in normal and saline-injected skin before and after losmapimod treatment. The top 30 genes upregulated in saline-injected skin as compared to normal skin before losmapimod treatment. d, Fold change of CCL2 ${ }^{+}$cells ( $n=7$ biologically independent individuals). e, Percentage of FSP1+CCL2+ cells that expressed p16 in saline-injected skin before and after losmapimod treatment ( $n=7$ biologically independent individuals). f, Fold change CD14 ${ }^{+}$cells in the skin following saline injection ( $n=9$ biologically independent individuals). $\mathbf{g}$, The number of CD14+ monocytes in VZV-injected skin ( 6 h after injection; $n=5$ biologically independent individuals) before and after losmapimod treatment. Significance in $\mathbf{d}$-g was assessed using a paired $t$-test. 
losmapimod treatment (Fig. 5c). This response to saline was reduced after pretreatment with losmapimod (Fig. 5c), and the top six significantly upregulated genes in saline-injected skin were no longer significantly different after losmapimod treatment, as compared to normal skin (Extended Data Fig. 10a). In addition, there was a strong trend towards a reduction in saline-specific gene expression after losmapimod treatment $(P=0.06$; Extended Data Fig. 10b). RNA-seq analysis showed that the expression of monocyte chemoattractants (induced in response to saline injection) was reduced after losmapimod treatment (Extended Data Fig. 10e). However, the reduction in genes specific to saline response in older participants (saline-specific gene expression) did not correlate with improvement a

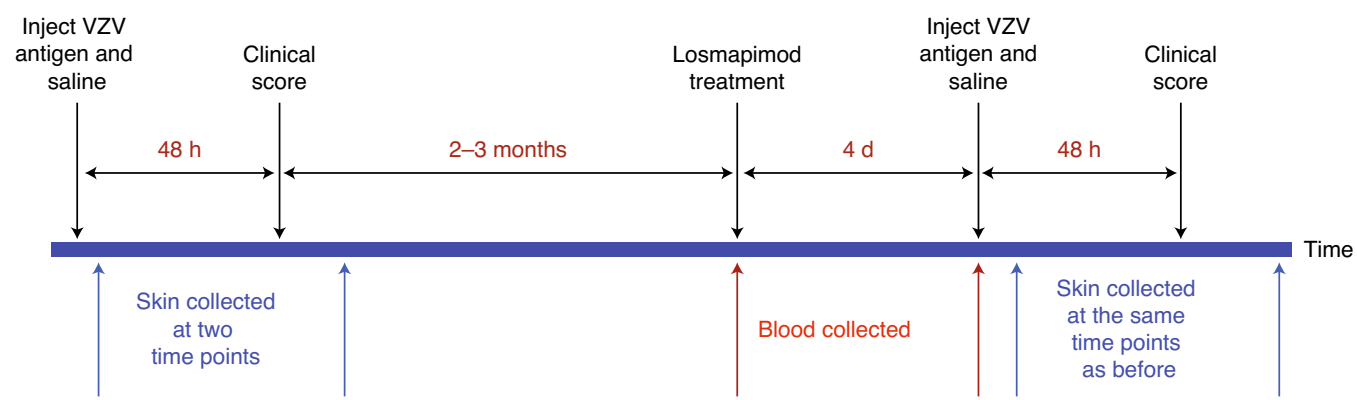

b

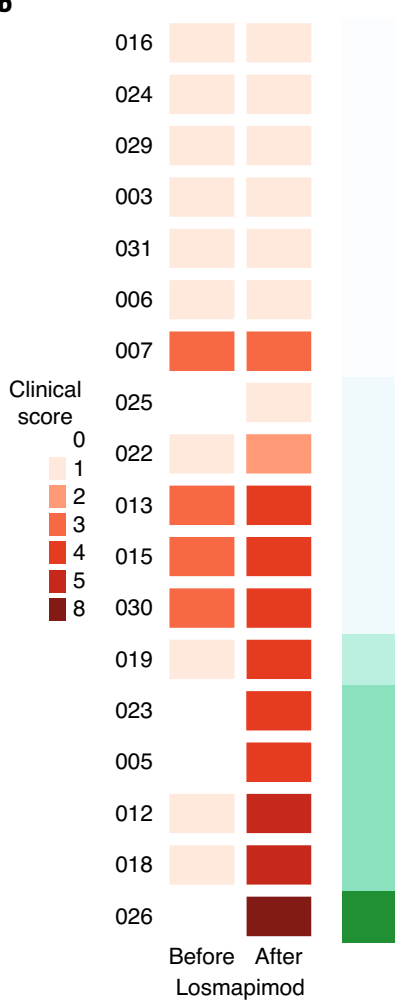

d

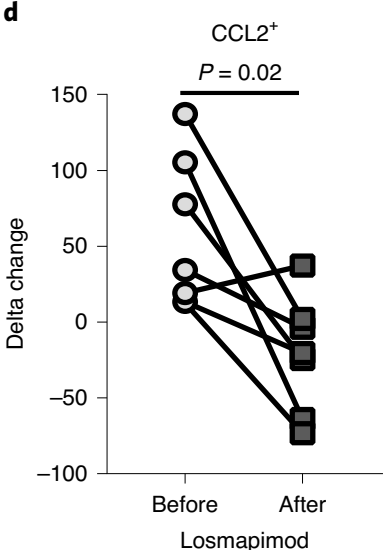

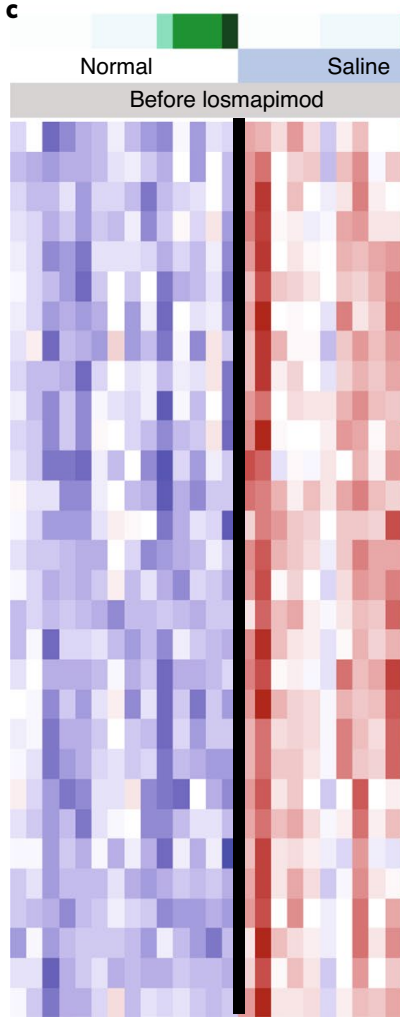

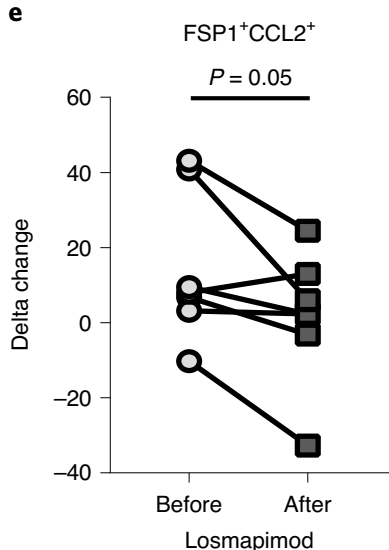

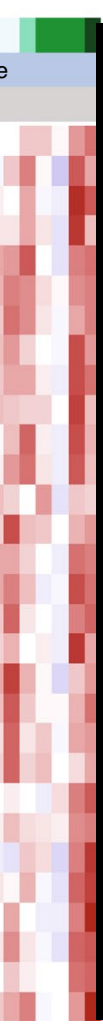

$\mathbf{f}$
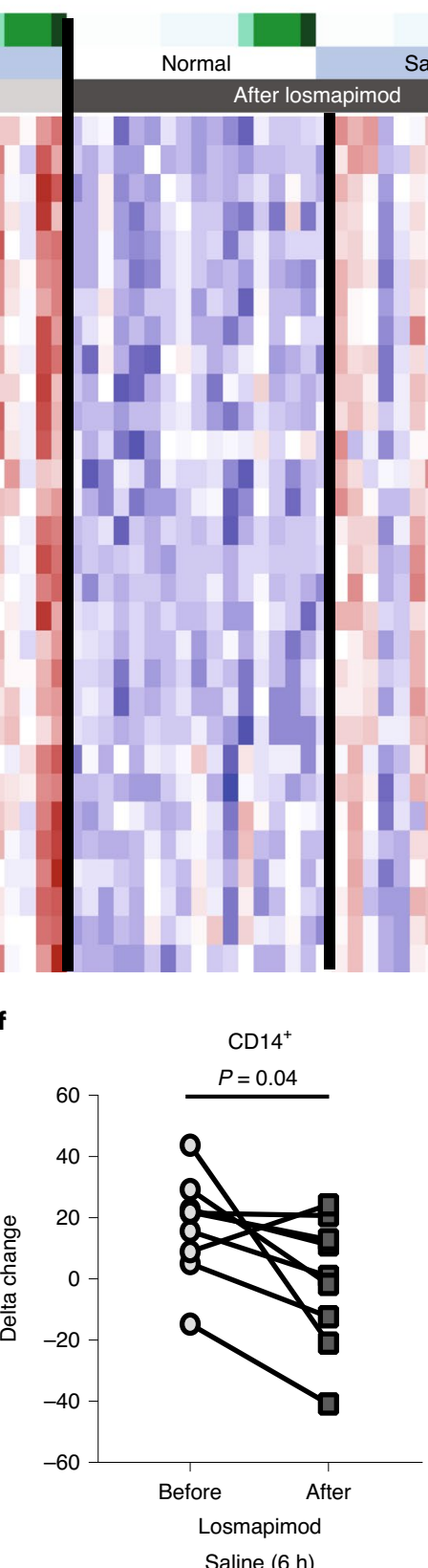

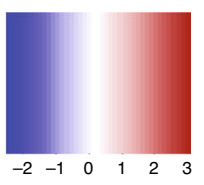
SERPINE1 FOSL THBS1 NR4A3 SOCS3 CCL2 RGS16 TNFAIP6 CXCL2 CXCL1 $\mathrm{CH} 25 \mathrm{H}$ TEX26-AS1 CRLF2 CCL8 SLC39A14 PTX3 FCGR1A ADAMTS8 MT1M ICAM1 NNMT $B T A F$ ITGAX CCR1 C5AR1 TREM1 SLC11A1 FCN1 SERPINA1 IL1B

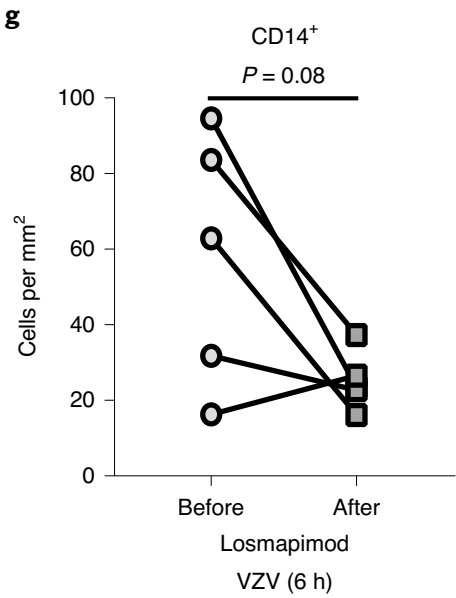




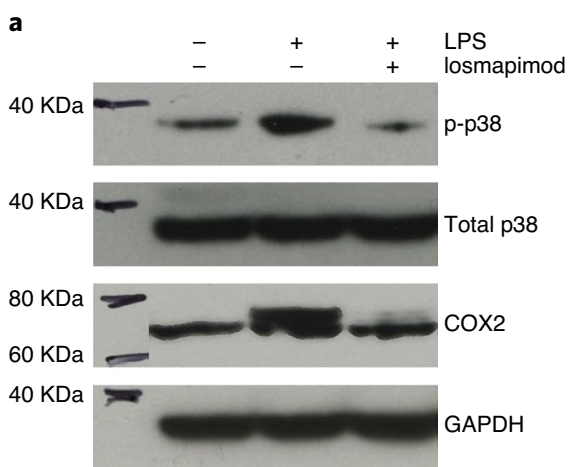

b

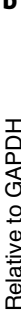

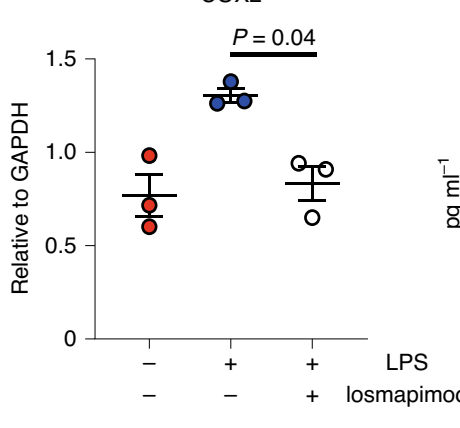

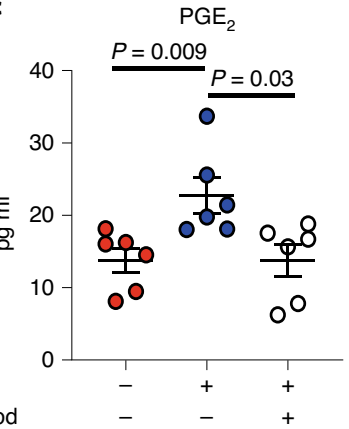
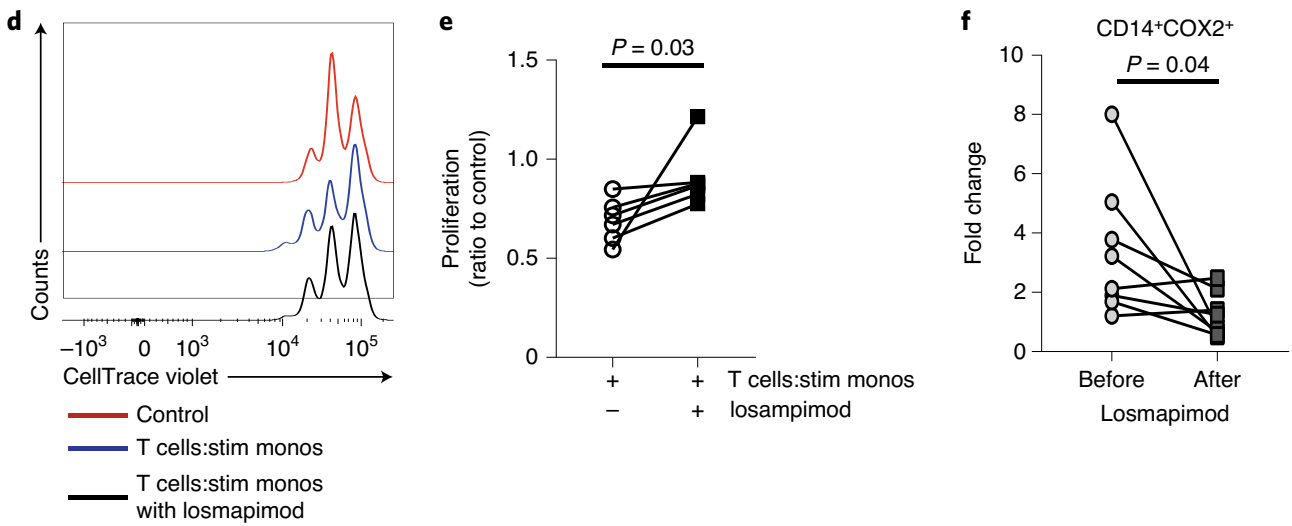

Fig. 6 | Losmapimod restores $T$ cell function by inhibiting monocyte COX2 and PGE 2 production. a-f, Monocytes were negatively isolated from the peripheral blood and cultured with and without LPS and in the presence or absence of losmapimod for $3 \mathrm{~h}$, pellets were collected and western blotting was performed. a, Representative blot of phospho-p38 MAPK (p-p38), total p38 MAPK (p38), COX2 and GAPDH (full scans are available as source data). b. Cumulative data of COX2 expression assessed by western blot relative to GAPDH $(n=3)$. c, PGE 2 production $(n=6)$. Monocytes were negatively isolated from the peripheral blood and stimulated with LPS (blue) and without LPS (red) or with LPS in the presence of losmapimod (white) for $3 \mathrm{~h}$ then subsequently co-cultured with autologous T cells (pre-labeled with CellTrace Violet) in the presence of plate-bound CD3. IL-2 proliferation was assessed at day 4. $\mathbf{d}$, A representative flow cytometry plot of CellTrace violet dilution in CD4+ $T$ cells co-cultured with unstimulated monocytes in the presence of losmapimod (control; red) or stimulated monocytes (blue) or stimulated monocytes in the presence of losmapimod (black). e, Cumulative data showing the percentage of divided cells $(n=6)$. f, Fold change of infiltration of $\mathrm{CD} 14^{+} \mathrm{COX} 2^{+}$cells between normal and saline-injected skin before and after losmapimod treatment $(n=8)$. Data show the mean \pm s.e.m., analyzed using one-way ANOVA with Tukey's multiple-comparison test $(\mathbf{b}$ and $\mathbf{c})$ and a Wilcoxon matched-pairs signed-rank test (e and $\mathbf{f})$.

in clinical score (Extended Data Fig. 10c), and the same was observed with CCL2 (Extended Data Fig. 10d).

After losmapimod treatment, there was a significant reduction in the number of $\mathrm{CCL}_{2}{ }^{+}$cells and the number of CCL2 ${ }^{+}$senescent $\left(\mathrm{p} 16^{+} \mathrm{FSP} 1^{+}\right)$fibroblasts (Fig. 5d,e). Treatment of senescent dermal fibroblasts with losmapimod in vitro significantly reduced their CCL2 production, suggesting losmapimod could have a direct effect on senescent fibroblasts in vivo (Extended Data Fig. 10f). As a result of the reduced CCL2 production, monocyte infiltration in saline-injected skin was significantly decreased after losmapimod treatment (Fig. 5f). A significant reduction in the number of monocytes after losmapimod treatment was observed when VZV-injected skin ( $6 \mathrm{~h}$ after injection) was assessed (Fig. $5 \mathrm{~g}$ ).

Together, these data indicate that p38 MAPK inhibition significantly reduces CCL2 production from senescent fibroblasts, reducing the accumulation of monocytes at sites of saline or VZV antigen challenge in the skin.

p38 MAPK inhibition significantly inhibits $\mathrm{PGE}_{2}$ that restores $\mathrm{T}$ cell proliferation. COX2 expression is shown to be regulated downstream of p38 MAPK signaling ${ }^{20,23}$, implying that losmapimod may act by inhibiting COX2 expression in vivo. To test this, we performed western blot analysis of LPS-activated monocytes in the presence or absence of losmapimod. Losmapimod significantly decreased LPS-induced expression of phospho-p38 MAPK and COX2 in vitro (Fig. 6a,b). In addition, $\mathrm{PGE}_{2}$ production was also significantly decreased after losmapimod treatment (Fig. 6c). Further, the addition of losmapimod to co-cultures of $\mathrm{CD} 4^{+} \mathrm{T}$ cells that were stimulated in the presence of LPS-treated monocytes (Fig. 6d,e and Supplementary Fig. 4c) enhanced their proliferation similar to that observed after direct COX2 or EP4 inhibition (Fig. $3 \mathrm{~g}, \mathrm{~h})$. In line with these observations, the increase in $\mathrm{CD} 14^{+} \mathrm{COX} 2^{+}$cells after saline injection was also inhibited after losmapimod treatment in vivo (Fig. 6f). Collectively, these data suggest that in vivo losmapimod can restore $\mathrm{CD} 4^{+} \mathrm{T}$ cell responses in part through the inhibition of COX2 expression.

p38 MAPK inhibition significantly increases $\mathrm{T}$ cell infiltration into the site of VZV challenge. We assessed the clinical score of 42 older participants after VZV antigen challenge before and after losmapimod treatment. We confirmed and extended our previous observations that treatment with losmapimod significantly increased VZV clinical score $(P<0.0001$; Fig. 7a). No difference was observed between male and female volunteers in their response to losmapimod treatment (Extended Data Fig. 1b). Losmapimod treatment also significantly decreased serum CRP in all participants, 
a

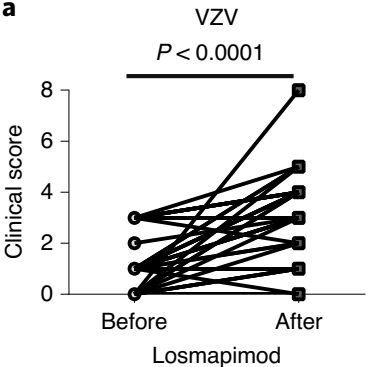

b

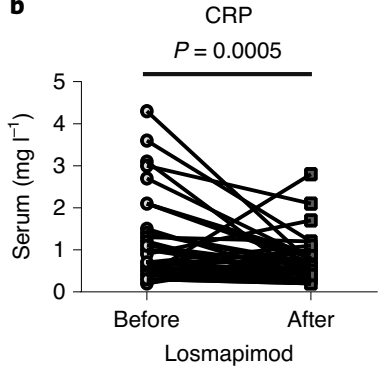

c
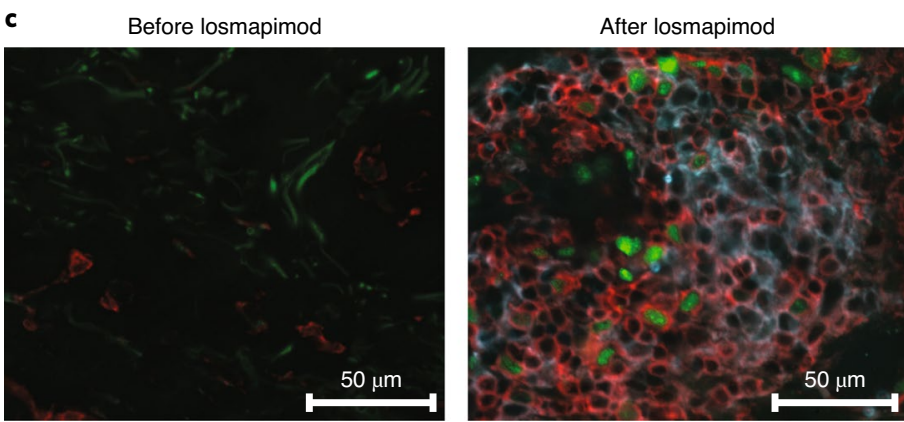

CD4; Ki67; CD110

d

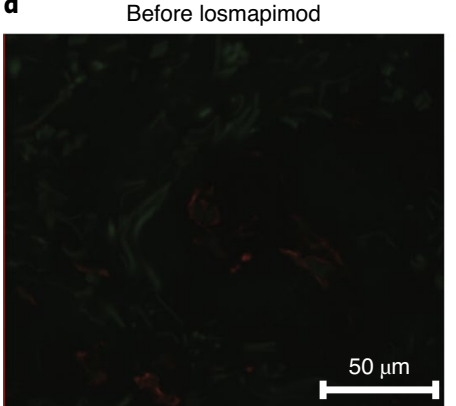

CD8; Ki67; CD11
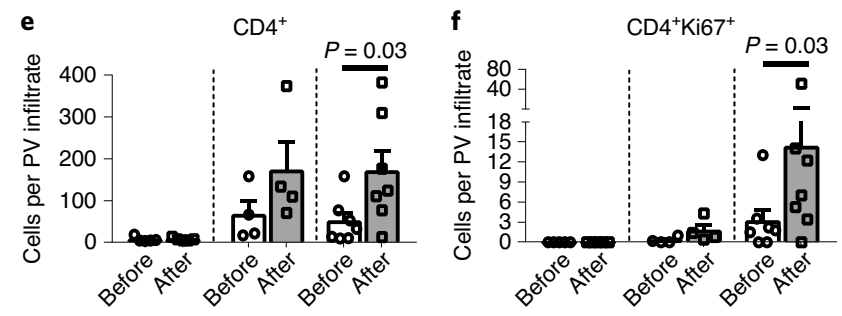

$6 \mathrm{~h} \quad 48 \mathrm{~h} \quad$ Day 7

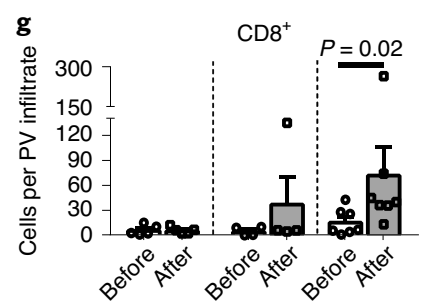

$6 \mathrm{~h} \quad 48 \mathrm{~h} \quad$ Day 7

$\mathrm{CD}_{11 \mathrm{C}^{+}}$

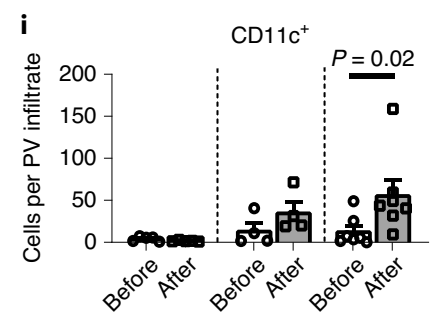

Fig. 7 | Losmapimod treatment increases VZV-induced T cell responses in the skin. $\mathbf{a}, \mathbf{b}$, VZV clinical score (a) and serum CRP concentrations (b) in all donors before and after losmapimod treatment $(n=42)$. c,d, Representative images of CD11c (blue) and Ki67 (green) and CD4 (red; c) or CD8 (red; d) staining at $7 \mathrm{~d}$ after VZV challenge before and after losmapimod. e-i, Cumulative data from CD4+ (e), CD4 $4^{+} \mathrm{Ki} 67^{+}(\mathbf{f}), \mathrm{CD}^{+}(\mathbf{g}), \mathrm{CD}^{+} \mathrm{Ki} i 7^{+}(\mathbf{h})$ and $\mathrm{CD}_{11 \mathrm{c}^{+}}(\mathbf{i})$ staining after $\mathrm{VZV}$ challenge in responders before and after losmapimod ( $6 \mathrm{~h}: n=5 ; 48 \mathrm{~h}: n=4$; day 7: $\left.n=7\right)$. Data in $\mathbf{e}-\mathbf{i}$ are shown as the mean \pm s.e.m. and were analyzed by a Wilcoxon matched-pairs test. PV, perivascular.

irrespective of whether they showed an improved skin response to VZV challenge (Fig. 7b).

We previously identified a significant association between the clinical score after VZV antigen challenge and the accumulation of $\mathrm{T}$ cells and DCs at the site of injection ${ }^{8,9}$. VZV-challenged skin sections collected before and after losmapimod were stained for CD4 or CD8, Ki67 and CD11c (Fig. 7c,d). We found that losmapimod pretreatment was associated with a significant increase in $\mathrm{CD}^{+}$(Fig. 7e) and $\mathrm{CD}^{+}$(Fig. $7 \mathrm{~g}$ ) $\mathrm{T}$ cell accumulation in the skin $7 \mathrm{~d}$ after VZV injection. The increased number of $\mathrm{CD} 4^{+}$and $\mathrm{CD} 8^{+}$ $\mathrm{T}$ cells in perivascular infiltrates was associated with an increased proliferation of these cells in situ, indicated by Ki67 expression (Fig. 7f,g) ${ }^{12}$. Concurrently, there was an increase in CD11 $\mathrm{c}^{+} \mathrm{DCs}$ in VZV-injected skin after losmapimod treatment (Fig. 7i), suggesting that there was an increase in T cell and DC clusters, as observed previously ${ }^{9}$. Taken together, our data show that short-term blockade of inflammation using the p38 MAPK inhibitor losmapimod can significantly enhance the cutaneous immune response to antigen challenge in older adults.

\section{Discussion}

We demonstrate that senescent fibroblasts in the skin of older participants secrete an array of chemokines including CCL2 in response to tissue injury, such as that induced by injection of saline, VZV or air. This leads to recruitment of inflammatory monocytes into the skin, which inhibit $\mathrm{T}_{\mathrm{RM}}$ cell activation in part by secreting lipid mediators such as $\mathrm{PGE}_{2}$ via a COX2-dependant pathway. However, short-term systemic p38 MAPK blockade (which also inhibits COX2) leads to a significant reduction in CCL2, infiltrating monocytes and reduced $\mathrm{PGE}_{2}$ expression in response to injection of saline or VZV antigens. This results in significant improvement of VZV-specific cutaneous T cell responses (Fig. 8). 

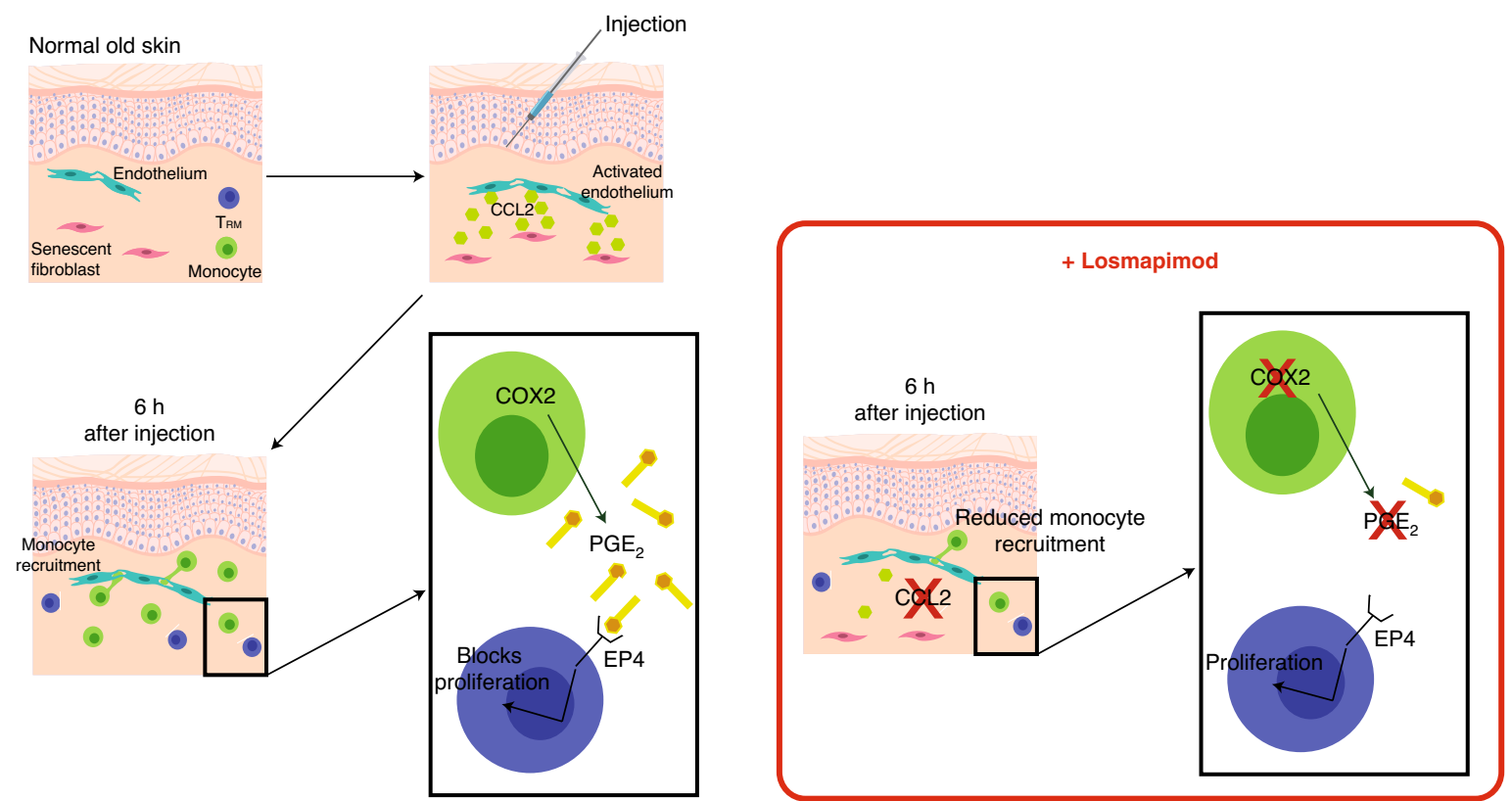

Fig. 8 | Schematic showing monocyte inhibition of cutaneous immunity in older adults. In older adults, the mild damage induced by injection (air, saline or VZV) resulted in production of CCL2 from senescent fibroblasts and recruitment of monocytes into the skin $6 \mathrm{~h}$ after injection. The recruited monocytes inhibited $T_{R M}$ cell proliferation via upregulation of $C O X 2$ and subsequent production of the lipid mediator PGE $_{2}$. The treatment of older adults with losmapimod resulted in reduced CCL2 production from the senescent fibroblasts and reduced monocyte infiltration. In addition, p38 MAPK was upstream of COX2 signaling; therefore, the treatment with losmapimod also inhibited COX2 upregulation and reduced PGE ${ }_{2}$ production. This significantly increased the T cell response to VZV in the skin of older participants.

Aging leads to the accumulation of senescent cells in many tissues including the $\operatorname{skin}^{16,18}$. Therefore, the recruitment of inflammatory monocytes by senescent fibroblasts may also contribute to age-related decreased immune responses in other organs. The significant correlation between the number of senescent dermal fibroblasts and the extent of CD $14^{+}$monocyte infiltration indirectly supports the interaction between these cells. Although the abundance of senescent cells increases in the skin during aging ${ }^{16-18}$, there is no overt expression of inflammatory mediators in unmanipulated skin of older participants ${ }^{12}$. This suggests that, in the steady state, the propensity to mount an inflammatory SASP response is inhibited by the increased numbers of Foxp $3^{+}$regulatory $\mathrm{T}$ cells and PD-1-expressing $\mathrm{T}_{\mathrm{RM}}$ cells observed in the skin of older people $\mathrm{e}^{10,12}$. Thus, the breakdown of homeostasis induced by tissue injury may override preexisting inhibitory processes.

$\mathrm{PGE}_{2}$ has disparate roles in immune responses as it has been shown to be pro-inflammatory (causing edema and pain), as well as contributing to resolution of inflammation ${ }^{22}$. $\mathrm{PGE}_{2}$ can also suppress $\mathrm{CD}^{+} \mathrm{T}$ cells and may delay viral clearance ${ }^{24}$. Here we show that $\mathrm{PGE}_{2}$ inhibits $\mathrm{CD}^{+} \mathrm{T}$ cell and $\mathrm{T}_{\mathrm{RM}}$ cell proliferation in vitro, thus confirming previous data ${ }^{25}$. Furthermore, $\mathrm{PGE}_{2}$ has been shown to induce the expansion and increase the function of Foxp $3^{+}$ regulatory $\mathrm{T}$ cells $\mathrm{s}^{26,27}$, which may explain why these cells increase in the skin during aging ${ }^{10,28}$. In addition, $\mathrm{PGE}_{2}$ may promote immunosuppression by increasing expression of the anti-inflammatory cytokine IL-10 by mononuclear phagocytes ${ }^{29,30}$. $\mathrm{PGE}_{2}$ can also have negative effects on $\mathrm{DCs}^{31}$. As CD11c $\mathrm{c}^{+} \mathrm{DCs}$ increase in the skin after losmapimod treatment, we cannot rule out that $\mathrm{PGE}_{2}$ may also have an inhibitory effect on DCs in the skin.

We have confirmed previous observations that monocytes can inhibit antigen-specific responses ${ }^{32,33}$. This may be relevant for the severe acute respiratory syndrome coronavirus 2 pandemic, which disproportionately affects older people ${ }^{34}$, since inflammatory monocytes have been found in the lungs of patients with severe coronavirus disease 2019 (ref. ${ }^{35}$ ) and these cells may inhibit the function of virus-specific T cells, as described above.

Excessive inflammation has been shown to inhibit antigenspecific immunity ${ }^{36}$ and reduce vaccination efficacy in many different contexts ${ }^{37-39}$. In addition to p38 MAPK inhibition, other drugs such as mammalian target of rapamycin inhibitors have been used to enhance immunity after vaccination in older adults ${ }^{40}$. Furthermore, COX2 inhibitors have shown promise at improving DC-based vaccine efficacy in mouse models of cancer ${ }^{41}$. The data presented here suggest that COX2 inhibitors, which are routinely used in other inflammatory conditions, could be used to improve immunity in older people. Thus, the inhibition of age-associated inflammation in older people may be a promising approach for enhancing immunity to infection, cancer therapy and vaccination during aging.

\section{Methods}

Study design. The investigation of healthy, young ( $<40$ years) and old ( $\geq 65$ years) participants was approved by the Queen Square Research Ethics Committee (London) and by the University College London (UCL) Institutional Review Board). Individuals with a history of neoplasia, immunosuppressive disorders or inflammatory skin disorders were excluded, as described elsewhere ${ }^{9}$ (see Supplementary Table 1 for donor characteristics). In brief, we excluded individuals with comorbidities that are associated with serious internal organ or immune dysfunction, including heart failure, severe chronic obstructive pulmonary disease, diabetes mellitus and rheumatoid arthritis, and individuals on immunosuppressive regimes for the treatment of autoimmune or chronic inflammatory diseases (for example, oral glucocorticoids, methotrexate, azathioprine and cyclosporin). In addition, individuals who had a history of liver disease or elevated liver transaminases ( $>1.5$ times the upper limit of normal) or an abnormal electrocardiogram were excluded. All volunteers provided written informed consent, and study procedures were performed in accordance with the principles of the Declaration of Helsinki. No statistical methods were used to predetermine sample sizes, but our sample sizes are similar to those reported in previous publications ${ }^{8,9,12,28}$.

For the study involving losmapimod, we recruited 42 healthy older adults (>65 years): 19 males and 23 females with a mean age of 70.7 (95\% CI: 69-72.3) 
years. A power calculation was performed to establish the effect on a change in clinical score, and we assumed an alpha level of 0.05 (two-sided) and a power of 0.90 was considered desirable. VZV skin tests were performed and biopsies collected at different time points as described previously ${ }^{9}$. Only individuals of European ancestry were included in the study, and any individual with a clinical score $>3$ was excluded from the study as described previously ${ }^{9}$. Two to three months after the first VZV skin challenge, the same volunteers received $15 \mathrm{mg}$ losmapimod (GW856553) twice a day for $4 \mathrm{~d}$ (provided by GlaxoSmithKline (GSK) under a Medical Research Council Industrial Collaboration Agreement). Previously, we found that the first VZV challenge did not significantly boost the response to rechallenge of the same individuals after 2-3 months ${ }^{9}$. The dosage (losmapimod $15 \mathrm{mg}$; twice daily) used in this study was chosen on the basis of the pharmacokinetic, pharmacodynamic and safety profiles of losmapimod observed in GSK Phase I and II studies ${ }^{42}$. These individuals were then rechallenged with VZV skin antigen, and biopsies were collected at the same time points as those before losmapimod treatment. Clinical scores after VZV injection were performed by two independent researchers, and an average reading was recorded. Serum CRP levels were measured with a high sensitivity assay (before and after losmapimod). No adverse side effects were observed in our study group in response to losmapimod pretreatment for $4 \mathrm{~d}$.

We were advised by the Medicines and Healthcare products Regulatory Agency that this study was not a clinical trial of an investigational medicinal product as defined by the EU Clinical Trials Directive (2001/20/EC). As this experimental medicine study was designed to test a hypothesis in humans in vivo and not to determine the therapeutic outcome or efficacy of losmapimod for patient benefit. Therefore, no randomization was performed.

Skin tests and punch biopsy. VZV antigen (BIKEN, The Research Foundation for Microbial Diseases of Osaka University) or $0.9 \%$ saline solution or an equal volume of air was injected intradermally into skin unexposed to the sun of the medial proximal volar forearm, per the manufacturer's instructions. Induration, palpability and the change in erythema from baseline were measured and scored on day 2 or 3 as described previously ${ }^{11}$. A clinical score (range 0-10) based on the summation of these parameters was then calculated. Donor characteristics can be found in Supplementary Table 1.

Two punch biopsies ( $5 \mathrm{~mm}$ in diameter) were collected from normal (unmanipulated) skin and either saline-injected or VZV-injected sites (at the indicated time points). Biopsies were frozen in optimal cutting temperature compound (Bright Instrument Company). Sections $(6 \mu \mathrm{m})$ were cut and left to dry overnight and then fixed in ethanol and acetone and stored at $-80^{\circ} \mathrm{C}$.

Immunofluorescence. Sections were stained with optimal dilutions of primary antibodies and followed by relevant secondary antibodies conjugated to various fluorochromes (Supplementary Table 2). Skin sections were imaged on the Axio Scan.Z1 slide scanner and analyzed using Zen Blue (Zeiss). For endothelial cell counts from normal skin and at $6 \mathrm{~h}$ after injection, the total cells in the upper dermis were counted and the number of cells per $\mathrm{mm}^{2}$ was calculated. Images were taken from the upper dermis (Fig. 1). For VZV-injected skin (days 2-7), the number of cells in five of the largest perivascular infiltrates present in the upper and mid-dermis was selected for analysis, and the average number was calculated as described previously ${ }^{10}$. Staining for $\mathrm{\gamma H}_{2} \mathrm{AX}$ TAF was carried out as described previously ${ }^{16}$. Counting was performed manually, and the data were blinded to the assessor to avoid any bias. Where multiple skin sections from one sample were assessed, the average of the counts was used.

Skin biopsy digestion for flow cytometric analysis. Skin biopsies ( $5 \mathrm{~mm})$ were taken from normal and saline-injected skin (6 or $24 \mathrm{~h}$ after injection) and disaggregated by overnight incubation $\left(37^{\circ} \mathrm{C}\right)$ in either $0.8 \mathrm{mg} \mathrm{ml}^{-1}$ collagenase IV (Sigma-Aldrich) with $20 \%$ FCS or a whole skin digestion kit (Miltenyi Biotec). Single-cell suspensions were obtained and subsequently stained with the cell surface antibodies listed in Supplementary Table 3. Samples were assessed by flow cytometric analysis on a BD Fortessa cell analyzer using FACSDiva software and subsequently analyzed using FlowJo version X (BD Biosciences).

Unbiased representations of multiparameter flow cytometry data were generated using the $t$-distributed stochastic neighbor embedding $(t$-SNE) algorithm. The R package 'Rtsne' available on CRAN (https://github.com/jkrijthe/ Rtsne/) was used to perform the Barnes Hut implementation of $t$-SNE on flow cytometry data. FlowJo software was used to export events of interest (in FCS format) for each sample. After using the Bioconductor 'flowCore' R package to import FCS file data and the Logicle transform to scale the data similarly to those displayed in FlowJo, 3,000 HLA-DR ${ }^{+}$events from each of the samples analyzed in parallel were merged and the relevant fluorescent parameters used.

RNA-seq analysis of skin. Three 3-mm punch biopsies were collected from each volunteer as follows: VZV injection site at $48 \mathrm{~h}$ after injection, saline injection site at $6 \mathrm{~h}$ after injection and normal (uninjected) skin as a control. Biopsies were immediately frozen in RNAlater. Frozen tissue was homogenized, and total RNA was extracted from bulk tissue homogenates using RNeasy Mini Kit (QIAGEN). Library preparation for RNA-seq was performed using the Kappa Hyperprep kit
(Roche Diagnostics), and sequencing was performed by the Pathogens Genomic Unit (UCL) on the Illumina NextSeq 500 using the NextSeq 500/550 High Output 75 cycle kit (Illumina) according to the manufacturer's instructions, providing 15-20 million 41-bp paired-end reads per sample. Reads were aligned to the human reference genome (GRCh38) using HISAT2 (ref. ${ }^{43}$ ). SAMtools was used to select for reads with paired mates. Transcript assembly was carried out using StringTie ${ }^{44}$, with gene-level fragments per kilobase of transcript per million mapped read (FPKM) generated using Ballgown ${ }^{45}$

Statistical comparisons were made on estimated gene counts generated by StringTie. Genes with low expression ( $<1$ count per million mapped reads in $95 \%$ of the samples) or short transcript lengths ( $<200$ nucleotides for the longest transcript) were removed. The count matrix was normalized using the trimmed mean of $\mathrm{M}$ values method in edgeR (version 3.22.5) ${ }^{46}$, followed by contrast.fit with voom in limma (version 3.36.5) ${ }^{47}$. The participant ID was used as a blocking variable. Statistically significant differentially expressed genes were identified as those with an adjusted $P$ value of less than 0.05 and expression change of greater than twofold.

Gene correlation networks were constructed and visualized using Graphia Professional (Kajeka, version 2.1; https://graphia.app/). Genes without a known HUGO gene nomenclature, or with expression < 1 FPKM for all samples, were removed from the coexpression network analysis. The Markov cluster algorithm was used to define the network clusters using an inflation value of 2.2. Saline-specific gene expression was generated using the average expression (FPKM) for genes that were significant before losmapimod in saline-injected skin as compared to normal skin (fold change $>2$ and $P<0.05$ ).

Leukocyte isolation from peripheral blood. Peripheral blood mononuclear cells were isolated by density centrifugation using Ficoll-Paque (Amersham Biosciences). $\mathrm{CD} 4^{+} \mathrm{T}$ cells were purified by positive selection, and monocytes were isolated by negative selection according to the manufacturer's instructions (Miltenyi Biotec).

Monocyte and T cells co-culture. Monocytes $\left(1 \times 10^{6} \mathrm{ml}^{-1}\right)$ were cultured in the presence of LPS $\left(1 \mathrm{ng} \mathrm{ml}^{-1}\right)$ and/or NS-398 $(1 \mu \mathrm{M})$ at $37^{\circ} \mathrm{C}$ with $5 \% \mathrm{CO}_{2}$. Monocytes were collected $3 \mathrm{~h}$ after incubation and washed once to remove residual LPS. These cells were preincubated with the inhibitor NS-398 $(1 \mu \mathrm{M})$ or losmapimod $(3 \mu \mathrm{M})$ where indicated. $\mathrm{CD} 4^{+} \mathrm{T}$ cells were labeled with CellTrace Violet (Invitrogen) before culture according to the manufacturer's instructions. $\mathrm{CD}^{+} \mathrm{T}$ cells and monocytes (unstimulated or stimulated with LPS) were co-cultured at different ratios, in the presence of plate-bound CD3 $\left(1 \mu \mathrm{g} \mathrm{ml}^{-1}\right)$ and IL-2 $\left(50 \mathrm{IU} \mathrm{ml}^{-1}\right)$ and MF-498 $(1 \mu \mathrm{M})$ at $37^{\circ} \mathrm{C}$ with $5 \% \mathrm{CO}_{2}$ for $4 \mathrm{~d}$.

For experiments involving co-culture of monocytes with skin-resident $\mathrm{T}_{\mathrm{RM}}$ cells, two suction blisters were formed over uninjected skin, as described previously ${ }^{11}$. Cells were collected from the blister the following day and incubated on plastic for $3 \mathrm{~h}$, to remove mononuclear phagocytes. Cell suspensions (predominantly $\mathrm{CD} 4^{+} \mathrm{T}$ cells) were removed and labeled with CellTrace Violet and co-cultured with unstimulated or LPS-stimulated monocytes as described above.

T cell proliferation assessment. Samples were collected on day 4 after culture and stained for CD3 (UCHT1), CD4 (RPA-T4; BD Biosciences) and the Zombie NIR Fixable Viability Kit (BioLegend). Samples were assessed by flow cytometric analysis on a BD Fortessa using FACSDiva software, and subsequently analyzed using FlowJo version X (BD Biosciences).

Western blot analysis. Cell pellets were harvested and lysed with RIPA buffer (Sigma-Aldrich) supplemented with protease and phosphatase inhibitors (Cell Signalling) for $30 \mathrm{~min}$ on ice. Cell lysates were diluted in SDS sample buffer with reducing agent (NuPage, Life Technologies) and boiled for $5 \mathrm{~min}$ at $95^{\circ} \mathrm{C}$. Samples were separated by protein electrophoresis at $120 \mathrm{~V}$ for $2 \mathrm{~h}$ using $10 \%$ Bis-Tris precast gels (NuPage) and transferred overnight at $4{ }^{\circ} \mathrm{C}$ onto Hybond-P PVDF membranes (GE Healthcare). After blocking in ECL blocking agent (GE Healthcare), membranes were probed with primary antibodies overnight at $4{ }^{\circ} \mathrm{C}$. Primary antibodies used were COX2 (ab15191; Abcam), phospho-p38 MAPK (T180/Y182; 9211), p38 MAPK (9212) and GAPDH (2118; Cell Signalling). The membrane was washed and incubated with HRP-conjugated secondary antibodies (GE Healthcare; 1:4,000 dilution) for $1 \mathrm{~h}$ at room temperature. Antibodies were detected using the ECL detection kit (GE Healthcare). Before reprobing with different antibodies, membranes were stripped at room temperature with agitation using Restore stripping buffer (Thermo Scientific). Protein bands were quantified using Image J software. The integrated density of each band was normalized to GAPDH using the gel analysis function in ImageJ.

Dermal fibroblast isolation and culture. Dermal fibroblasts were isolated and cultured as described previously ${ }^{16}$. Senescence was induced in these cells by exposure to X-ray radiation at a total dose of $10 \mathrm{~Gy}$ at a rate of $5 \mathrm{~Gy} \mathrm{~min}^{-1}$. Irradiated cells were cultured for a further $10-28 \mathrm{~d}$ to allow for senescence to develop. Senescence was confirmed by staining for senescence-associated beta-galactosidase (Cell signalling). Non-senescent (early passage number: 4-10) and senescent fibroblasts were cultured in a 48 -well plate at $20 \times 10^{4}$ cells per well 
in the presence of absence of losmapimod $(3 \mu \mathrm{M})$, and supernatants were collected at 3 and $24 \mathrm{~h}$.

Assessment of supernatants. Cytokine concentrations in culture supernatants were assessed by cytometric bead array according to the manufacturer's protocol. Samples were analyzed using a BD Verse flow cytometer (BD Biosciences). The lower limit of detection for each analyte was $1.5 \mathrm{pg} \mathrm{ml}^{-1}$. For assessment of $\mathrm{PGE}_{2}$ production from monocyte cultures, a $\mathrm{PGE}_{2}$ Direct Biotrak Assay was used according to the manufacturer's protocol (Fisher Scientific).

Statistics. Statistical analysis was performed using Prism version 8.0.0 (GraphPad). Data were assessed for normality, and the subsequent appropriate two-sided statistical test was performed.

Reporting Summary. Further information on research design is available in the Nature Research Reporting Summary linked to this article.

\section{Data availability}

RNA-seq data that support the findings of this work have been deposited in the NCBI Gene Expression Omnibus under accession GSE130633. Source data are provided with this paper

\section{Code availability}

Scripts used in the RNA-seq analysis can be found at https://github.com/ barbarashih/arise_losmapimod/.

Received: 14 May 2020; Accepted: 25 November 2020; Published online: 14 January 2021

\section{References}

1. Gavazzi, G. \& Krause, K. H. Ageing and infection. Lancet. Infect. Dis. 2, 659-666 (2002).

2. Diffey, B. L. \& Langtry, J. A. Skin cancer incidence and the ageing population. Br. J. Dermatol. 153, 679-680 (2005).

3. Ciabattini, A. et al. Vaccination in the elderly: the challenge of immune changes with aging. Semin. Immunol. 40, 83-94 (2018).

4. Franceschi, C., Garagnani, P., Vitale, G., Capri, M. \& Salvioli, S. Inflammaging and 'Garb-aging'. Trends Endocrinol. Metab. 28, 199-212 (2017).

5. Furman, D. et al. Expression of specific inflammasome gene modules stratifies older individuals into two extreme clinical and immunological states. Nat. Med. 23, 174-184 (2017).

6. Dinh, K. M. et al. Low-grade inflammation is negatively associated with physical health-related quality of life in healthy individuals: results from The Danish Blood Donor Study. PLoS ONE 14, e0214468 (2019).

7. Chambers, E. S. \& Akbar, A. N. Can blocking inflammation enhance immunity during aging? J. Allergy Clin. Immunol. 145, 1323-1331 (2020).

8. Agius, E. et al. Decreased TNF-alpha synthesis by macrophages restricts cutaneous immunosurveillance by memory $\mathrm{CD}^{+} \mathrm{T}$ cells during aging. J. Exp. Med. 206, 1929-1940 (2009).

9. Vukmanovic-Stejic, M. et al. Enhancement of cutaneous immunity during aging by blocking p38 mitogen-activated protein kinase-induced inflammation. J. Allergy Clin. Immunol. 142, 844-856 (2018).

10. Vukmanovic-Stejic, M. et al. The kinetics of $\mathrm{CD}^{+}{ }^{+}$Foxp $3^{+} \mathrm{T}$ cell accumulation during a human cutaneous antigen-specific memory response in vivo. J. Clin. Invest. 118, 3639-3650 (2008).

11. Akbar, A. N. et al. Investigation of the cutaneous response to recall antigen in humans in vivo. Clin. Exp. Immunol. 173, 163-172 (2013).

12. Vukmanovic-Stejic, M. et al. The characterization of varicella zoster virus-specific T cells in skin and blood during aging. J. Invest. Dermatol. 135, 1752-1762 (2015).

13. Patel, A. A. et al. The fate and lifespan of human monocyte subsets in steady state and systemic inflammation. J. Exp. Med. 214, 1913-1923 (2017).

14. Ziegler-Heitbrock, L. et al. Nomenclature of monocytes and dendritic cells in blood. Blood 116, e74-e80 (2010).

15. Tamoutounour, S. et al. Origins and functional specialization of macrophages and of conventional and monocyte-derived dendritic cells in mouse skin. Immunity 39, 925-938 (2013).

16. Pereira, B. I. et al. Senescent cells evade immune clearance via HLA-E-mediated NK and CD8 ${ }^{+} \mathrm{T}$ cell inhibition. Nat. Commun. 10, 2387 (2019).

17. Dimri, G. P. et al. A biomarker that identifies senescent human cells in culture and in aging skin in vivo. Proc. Natl Acad. Sci. USA 92, 9363-9367 (1995)

18. Ressler, S. et al. p16INK4A is a robust in vivo biomarker of cellular aging in human skin. Aging Cell 5, 379-389 (2006).

19. Campisi, J. Aging, cellular senescence and cancer. Annu. Rev. Physiol. 75, 685-705 (2013).
20. Dean, J. L., Brook, M., Clark, A. R. \& Saklatvala, J. p38 mitogen-activated protein kinase regulates cyclooxygenase- 2 mRNA stability and transcription in lipopolysaccharide-treated human monocytes. J. Biol. Chem. 274, 264-269 (1999)

21. Mestre, J. R. et al. Redundancy in the signaling pathways and promoter elements regulating cyclooxygenase- 2 gene expression in endotoxin-treated macrophage/monocytic cells. J. Biol. Chem. 276, 3977-3982 (2001).

22. Kalinski, P. Regulation of immune responses by prostaglandin $\mathrm{E}_{2}$. J. Immunol. 188, 21-28 (2012)

23. Guan, Z., Buckman, S. Y., Pentland, A. P., Templeton, D. J. \& Morrison, A. R. Induction of cyclooxygenase-2 by the activated MEKK1-SEK1/MKK4-p38 mitogen-activated protein kinase pathway. J. Biol. Chem. 273, 12901-12908 (1998)

24. Chen, $\mathrm{H}$. et al. Elevated COX2 expression and $\mathrm{PGE}_{2}$ production by downregulation of RXR $\alpha$ in senescent macrophages. Biochem. Biophys. Res. Commun. 440, 157-162 (2013).

25. Okano, M. et al. E prostanoid 2 (EP2)/EP4-mediated suppression of antigen-specific human T-cell responses by prostaglandin E2. Immunology 118, 343-352 (2006)

26. Baratelli, F. et al. Prostaglandin E2 induces FOXP3 gene expression and $\mathrm{T}$ regulatory cell function in human $\mathrm{CD} 4^{+} \mathrm{T}$ cells. J. Immunol. $\mathbf{1 7 5}$ 1483-1490 (2005).

27. Sharma, S. et al. Tumor cyclooxygenase-2/prostaglandin E2-dependent promotion of FOXP3 expression and $\mathrm{CD} 4{ }^{+} \mathrm{CD} 25^{+} \mathrm{T}$ regulatory cell activities in lung cancer. Cancer Res. 65, 5211-5220 (2005).

28. Vukmanovic-Stejic, M. et al. Varicella zoster-specific CD4 ${ }^{+} \mathrm{Foxp}^{+} \mathrm{T}$ cells accumulate after cutaneous antigen challenge in humans. J. Immunol. 190, 977-986 (2013)

29. Nakanishi, M. \& Rosenberg, D. W. Multifaceted roles of $\mathrm{PGE}_{2}$ in inflammation and cancer. Semin. Immunopathol. 35, 123-137 (2013).

30. MacKenzie, K. F. et al. $\mathrm{PGE}_{2}$ induces macrophage IL-10 production and a regulatory-like phenotype via a protein kinase A-SIK-CRTC3 pathway. J. Immunol. 190, 565-577 (2013).

31. Zelenay, S. et al. Cyclooxygenase-dependent tumor growth through evasion of immunity. Cell 162, 1257-1270 (2015).

32. Watanabe, R. et al. Pyruvate controls the checkpoint inhibitor PD-L1 and suppresses T cell immunity. J. Clin. Invest. 127, 2725-2738 (2017).

33. Sammicheli, S. et al. Inflammatory monocytes hinder antiviral B cell responses. Sci. Immunol. 1, eaah6789 (2016).

34. Chen, G. et al. Clinical and immunological features of severe and moderate coronavirus disease 2019. J. Clin. Invest. 130, 2620-2629 (2020).

35. Liao, M. et al. Single-cell landscape of bronchoalveolar immune cells in patients with COVID-19. Nat. Med. 26, 842-844 (2020).

36. Pence, B. D. et al. Relationship between systemic inflammation and delayed-type hypersensitivity response to candida antigen in older adults. PLoS ONE 7, e36403 (2012)

37. Fourati, S. et al. Pre-vaccination inflammation and B-cell signalling predict age-related hyporesponse to hepatitis B vaccination. Nat. Commun. 7, 10369 (2016).

38. Muyanja, E. et al. Immune activation alters cellular and humoral responses to yellow fever 17D vaccine. J. Clin. Invest. 124, 3147-3158 (2014).

39. Parmigiani, A. et al. Impaired antibody response to influenza vaccine in HIV-infected and uninfected aging women is associated with immune activation and inflammation. PLoS ONE 8, e79816 (2013).

40. Mannick, J. B. et al. mTOR inhibition improves immune function in the elderly. Sci. Transl. Med. 6, 268 ra179 (2014).

41. Hahn, T. et al. Short-term dietary administration of celecoxib enhances the efficacy of tumor lysate-pulsed dendritic cell vaccines in treating murine breast cancer. Int. J. Cancer 118, 2220-2231 (2006).

42. Watz, H., Barnacle, H., Hartley, B. F. \& Chan, R. Efficacy and safety of the p38 MAPK inhibitor losmapimod for patients with chronic obstructive pulmonary disease: a randomised, double-blind, placebo-controlled trial. Lancet Respir. Med. 2, 63-72 (2014).

43. Kim, D., Langmead, B. \& Salzberg, S. L. HISAT: a fast spliced aligner with low memory requirements. Nat. Methods 12, 357-360 (2015).

44. Pertea, M. et al. StringTie enables improved reconstruction of a transcriptome from RNA-seq reads. Nat. Biotechnol. 33, 290-295 (2015).

45. Frazee, A. C. et al. Ballgown bridges the gap between transcriptome assembly and expression analysis. Nat. Biotechnol. 33, 243-246 (2015).

46. Robinson, M. D., McCarthy, D. J. \& Smyth, G. K. edgeR: a Bioconductor package for differential expression analysis of digital gene expression data Bioinformatics 26, 139-140 (2010).

47. Ritchie, M. E. et al. limma powers differential expression analyses for RNA-sequencing and microarray studies. Nucleic Acids Res. 43, e47 (2015).

\section{Acknowledgements}

This work was funded by a Medical Research Council (MRC) Grand Challenge in Experimental Medicine grant (MICA; MR/M003833/1 to A.N.A., M.V.-S., T.C.F. and N.A.M.), an MRC New Investigator award (G0901102 to M.V.-S.), a Dermatrust 
and MRC responsive mode grant (MR/T030534/1 to A.N.A.), the British Skin Foundation (BSF5012 to A.N.A.), Institute Strategic Programme Grant funding from the Biotechnology and Biological Sciences Research Council (BBS/E/D/20002173 to T.C.F. and N.A.M.) and the National Institute for Health Research UCL Hospitals Biomedical Research Centre. We thank L. R. Glaser, L. Sarov-Blat and R. Henderson at GSK, V. Birault at the Francis Crick Institute and M. Noursadeghi at UCL for support in developing this project. We are also grateful to GSK for providing losmapimod (MICA agreement with the MRC) and to the losmapimod team at GSK for organizing the dispatch of the clinical supply of the drug in this investigator-led study. We especially thank the blood and skin donors who volunteered for this study and our research nurses M. Harries-Nee and M. Berkley for their outstanding work.

\section{Author contributions}

E.S.C. designed and performed experiments and wrote the manuscript. M.V.-S. was involved in the overall design of the study, experimental design and wrote the manuscript. H.T. and O.P.D. performed the experiments. B.B.S., N.A.M. and T.C.F. performed the bioinformatic analysis of the RNA-seq samples. P.S. and J.G. performed clinical procedures and sample collection. M.R. was the clinical lead for the study and was involved with scientific discussions. D.G. advised on experimental design for
COX2 and $\mathrm{PGE}_{2}$ work. A.N.A. was involved in the overall design of the study, initiated and coordinated the collaborative interaction between the different research groups, interpreted the data, and contributed to writing and editing of the manuscript.

\section{Competing interests}

The authors declare no competing interests.

\section{Additional information}

Supplementary information is available for this paper at https://doi.org/10.1038/ s43587-020-00010-6.

Correspondence and requests for materials should be addressed to A.N.A.

Peer review information Nature Aging thanks the anonymous reviewers for their contribution to the peer review of this work.

Reprints and permissions information is available at www.nature.com/reprints.

Publisher's note Springer Nature remains neutral with regard to jurisdictional claims in published maps and institutional affiliations.

(C) The Author(s), under exclusive licence to Springer Nature America, Inc. 2021 
A

CD14+

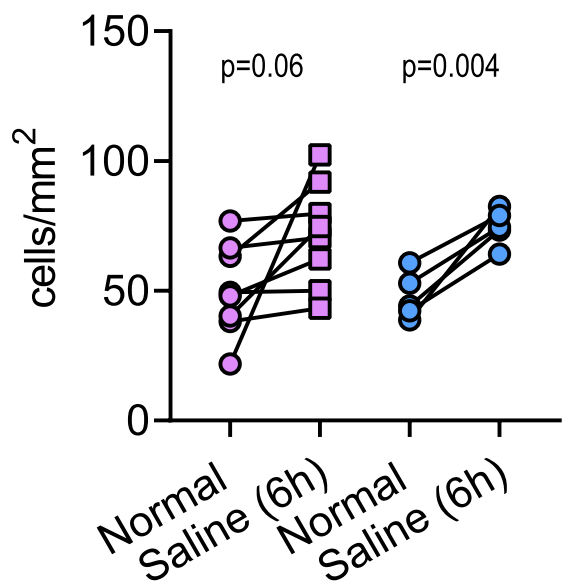

CCR2+

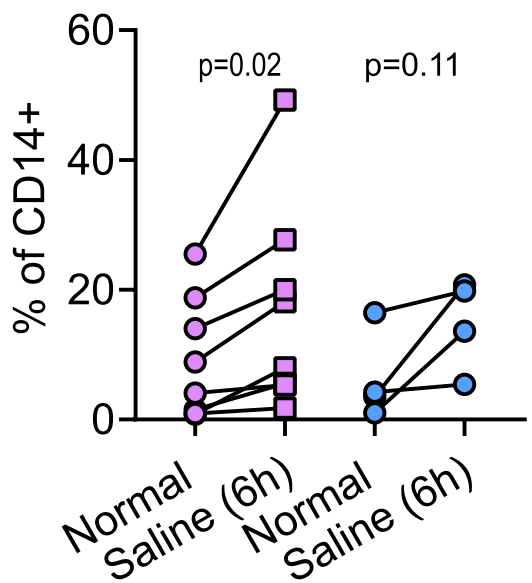

B

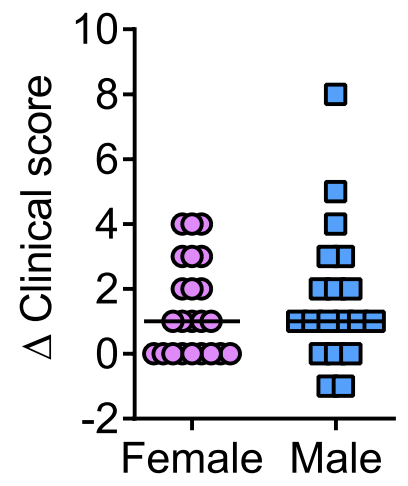

Extended Data Fig. 1 | No gender difference observed in older adults in response to saline or Losmapimod. A, cumulative data showing the number of $\mathrm{CD} 14+$ and frequency of CCR2 + CD14 + cells in females (pink; $n=8$ ) and males (blue; $n=5$ ). B, Change in clinical score after Losmapimod treatment in female (pink; $n=23$ ) and male (blue; $n=19$ ), line indicates median. Data assessed by paired $t$-test within donor and non-paired t test for comparison between female and males. 
A
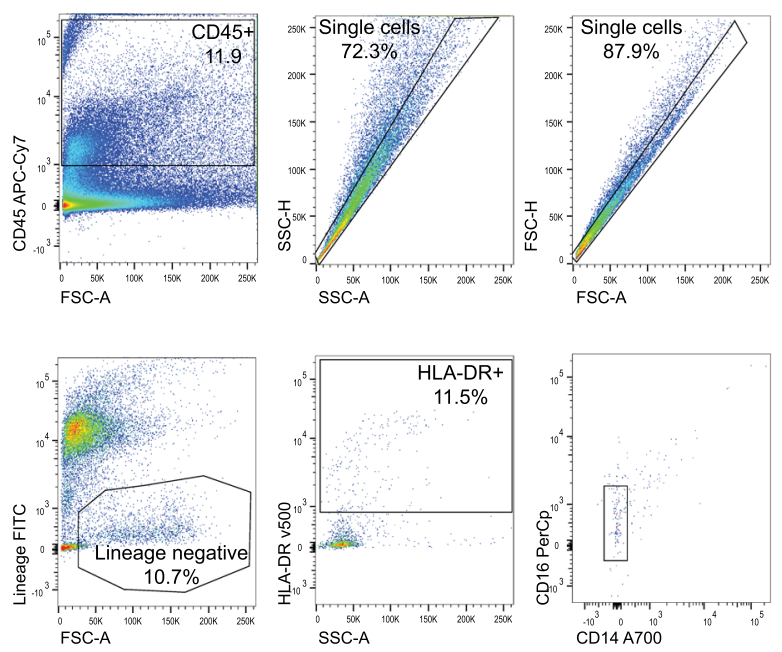
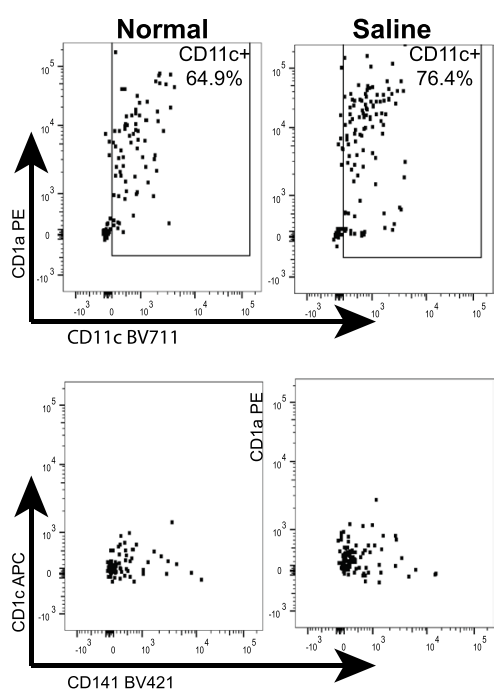

B
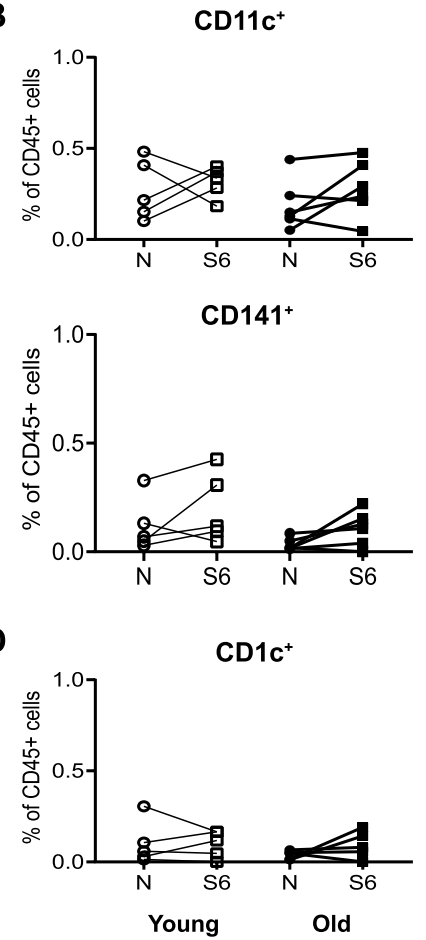

Extended Data Fig. 2 | Flow cytometric analysis of dendritic cell populations in normal and saline injected skin. A, Normal and Saline-injected skin were digested overnight and assessed the following day by multiparametric flow cytometry. CD45+ cells were identified, followed by two singlet gates, subsequently cells that were CD3, CD56, CD19, CD20 Zombie Green (Lineage) negative and HLA-DR + were selected, CD14-CD16-CD11c + cells were assumed to be dendritic cells. Within the CD11c + population CD1c and CD141 expression was assessed. Representative staining from Normal and Saline injected ( 6 hours) skin collected from an older adult is shown. Gating is based upon FMO controls from matched blood sample. Cumulative flow cytometry data of $B, C D 11 c+, C, C D 141+$ and D, CD1c + dendritic cells (young $n=5$; old $n=6$ biologically independent individuals). Data analysed by paired $t$ test and no significance was found. 
A

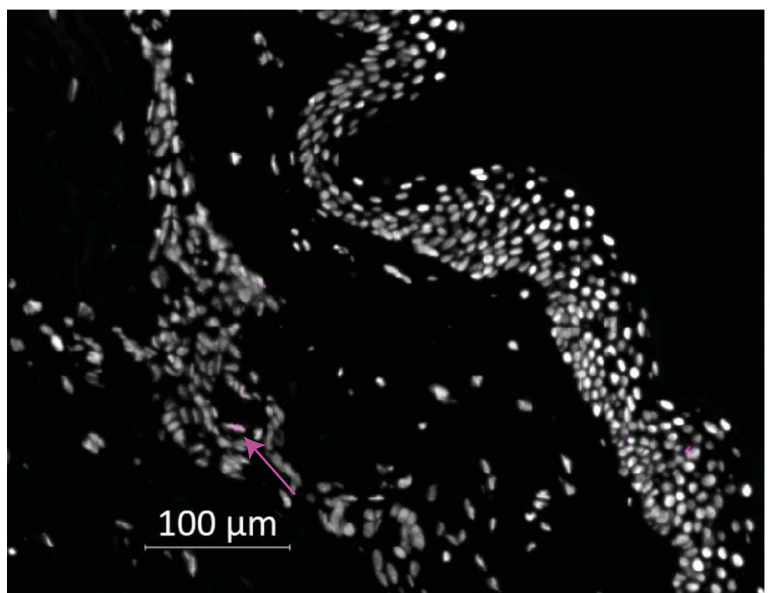

B

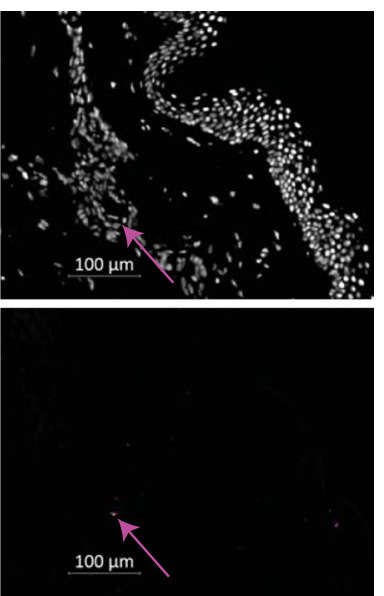

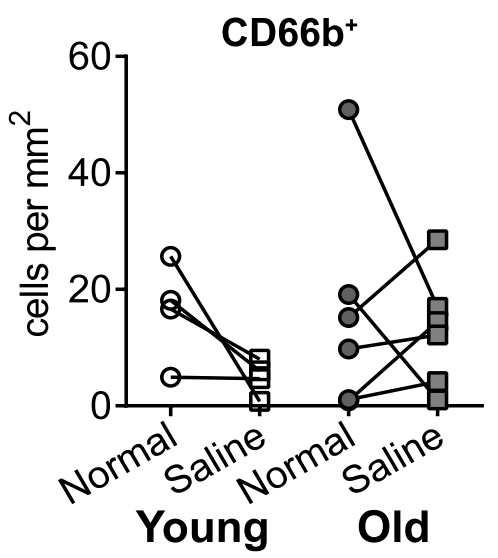

Extended Data Fig. 3 | No change in CD66b + neutrophil numbers after saline-injection. A, representative staining of saline-injected skin stained for DAPI (white), and CD66b (purple) and B, cumulative data showing the number of CD66b + cells in normal and saline-injected young (white) and old (grey) (young $n=4$; old $n=6$ biologically independent individuals). Data assessed by paired $t$-test and no significance was found. 
A

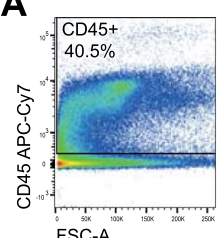

D

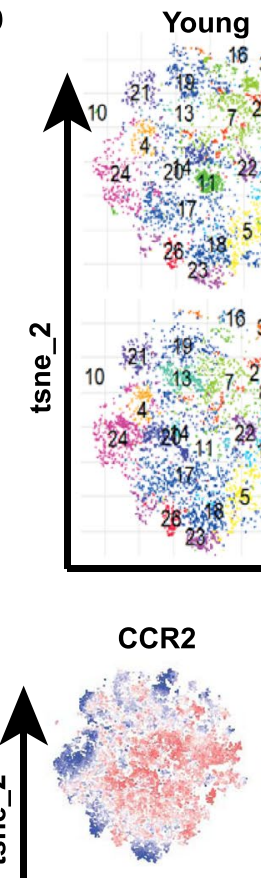

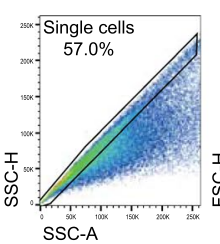
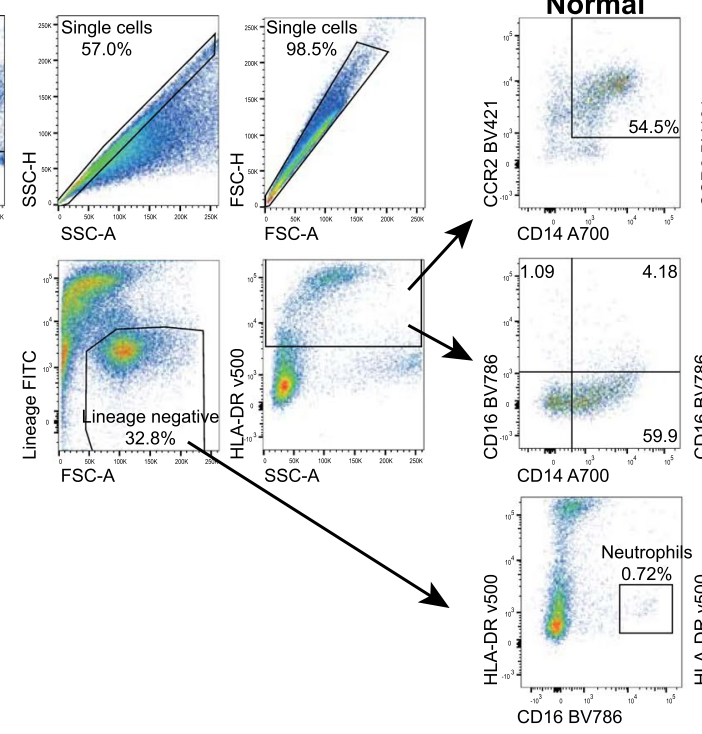

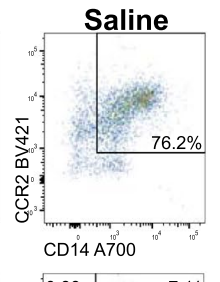

B

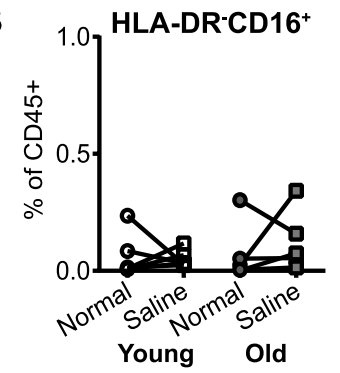

C

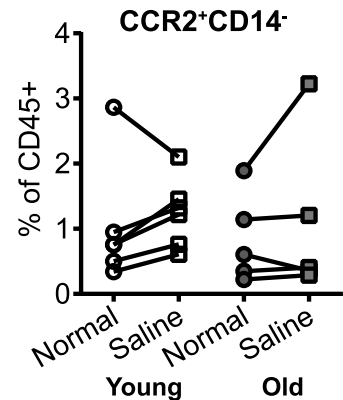

E

Normal
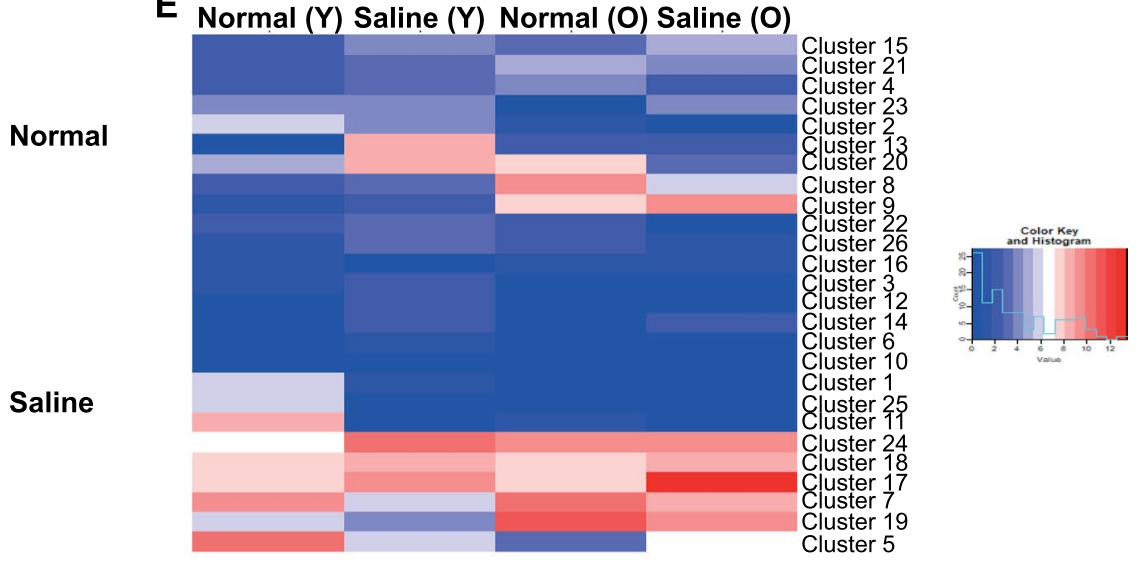

Saline

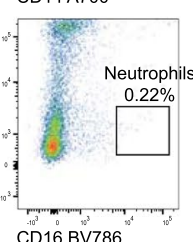

Old
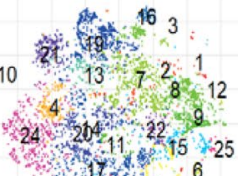
$\frac{26}{23}+5$
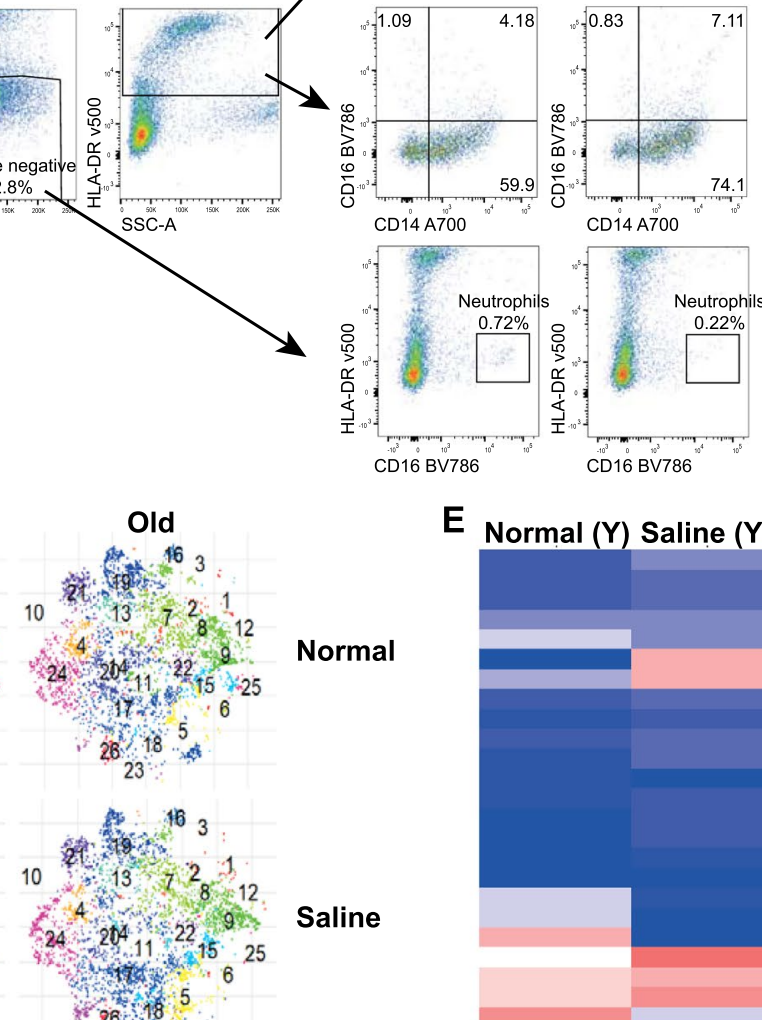

CD

Young

Old

CD14

CD16

CD86

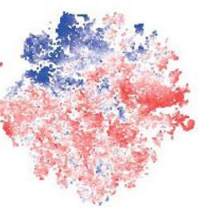

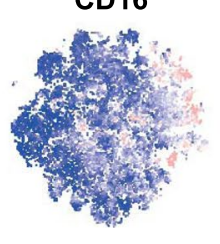

CLA

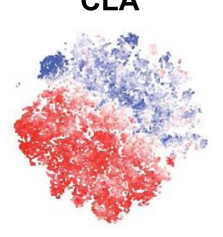

$\mathrm{CX}_{3} \mathrm{CR} 1$

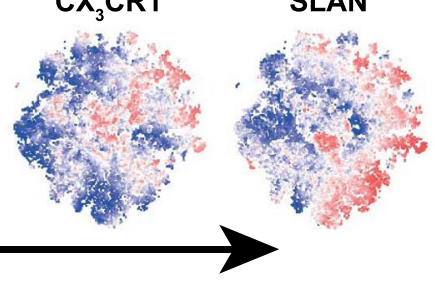

Extended Data Fig. 4 | Baseline and 24 hours post-saline injection monocyte populations. A, Normal and Saline-injected skin were digested overnight and assessed the following day by multiparametric flow cytometry. CD45 + cells were identified, followed by two singlet gates, subsequently cells that were CD3, CD56, CD19, CD20 Zombie Green (Lineage) negative and HLA-DR + were considered to be mononuclear phagocytes, within this population $\mathrm{CD} 14+\mathrm{CCR} 2+$ and CD14 + CD16 + monocytes and CD16 + HLA-DR - neutrophils were assessed. Representative staining from Normal and Saline injected (6 hours) skin collected from an older adult is shown. Gating is based upon FMO controls from matched blood sample. Normal and Saline-injected young (white) and old (grey) skin was assessed by flow cytometry and B, cumulative data of the frequency of CD16+HLA-DR- neutrophils and C, cumulative frequency of the number of CCR2 $+C D 14^{-}$cells (young $n=7$; old $n=6$ ). Unbiased representations of multi-parameter flow cytometry data were generated using the t-distributed stochastic neighbor embedding (tsne) algorithmt-SNE. Tsne is a non-linear dimensionality reduction method that optimally places cells with similar expression levels near to each other and cells with dissimilar expression levels further apart. $D$, tsne cluster plots of young and old normal and saline injected skin and $\mathrm{E}$, heatmap of clusters in normal and saline-injected skin of young $(\mathrm{Y})$ and old $(\mathrm{O})$ adults and $\mathrm{F}$, marker expression plots. Data analysed by paired t test and no significance was found. 
A

CD14+

CCR2+
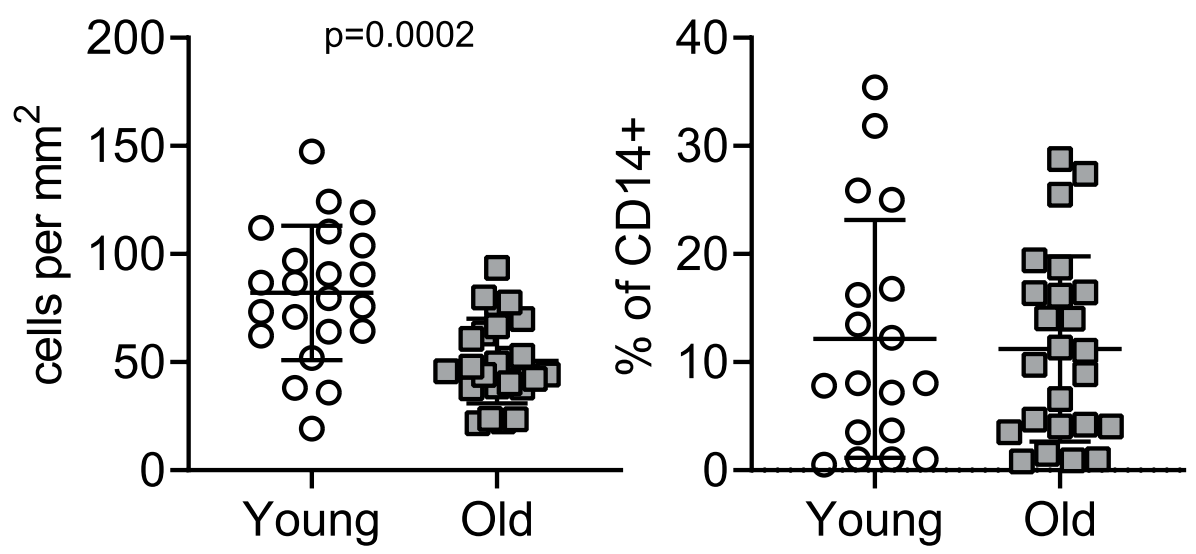

B

CD14+

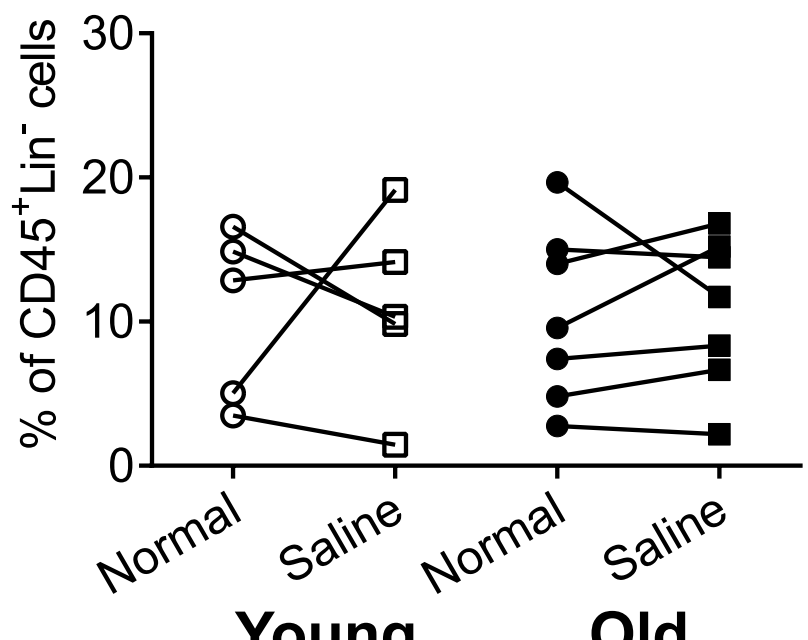

C

CD14 $^{+} \quad$ CCR2 $^{+}$CD14 ${ }^{+}$

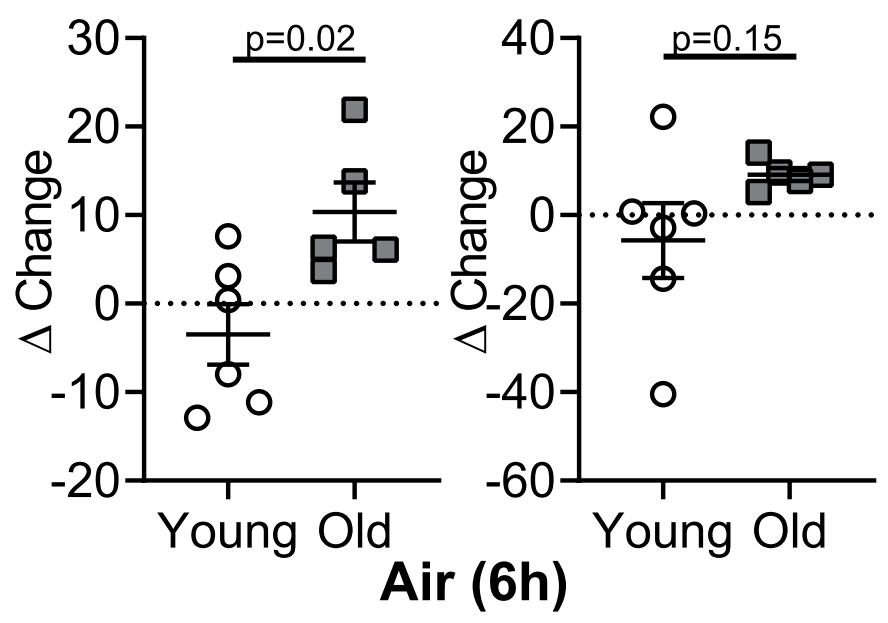

Extended Data Fig. 5 | See next page for caption. 
Extended Data Fig. 5 | Baseline and 24 hours post-saline injection monocyte populations. A, Comparison of baseline monocyte populations in the skin of based on CD14 + and CCR2 + expression in young ( $n=22$ biologically independent individuals) and old ( $n=24$ biologically independent individuals) individuals. B, Normal and Saline-injected skin were collected 24 hours post-injection from $n=5$ young (average age 30 years; 3 male and 2 females) and $n=7$ old (average age 70 years; 2 males and 5 females). Samples were digested overnight and assessed the following day by multiparametric flow cytometry. Cells that were CD45+HLA-DR + and CD3, CD56, CD19 and CD20 negative were considered to be mononuclear phagocytes, within this population CD14 + monocytes were assessed. C, Cumulative delta change in number of CD14 + or CCR2 + CD14 + cells in air-injected skin from young ( $n=6$ biologically independent individuals) and old ( $n=5$ biologically independent individuals) volunteers as compared to normal skin. $A$, and $C$, shown as mean \pm SEM. $A$ and $C$ assessed by an unpaired $t$ test and $B$, assessed by paired t-test. 

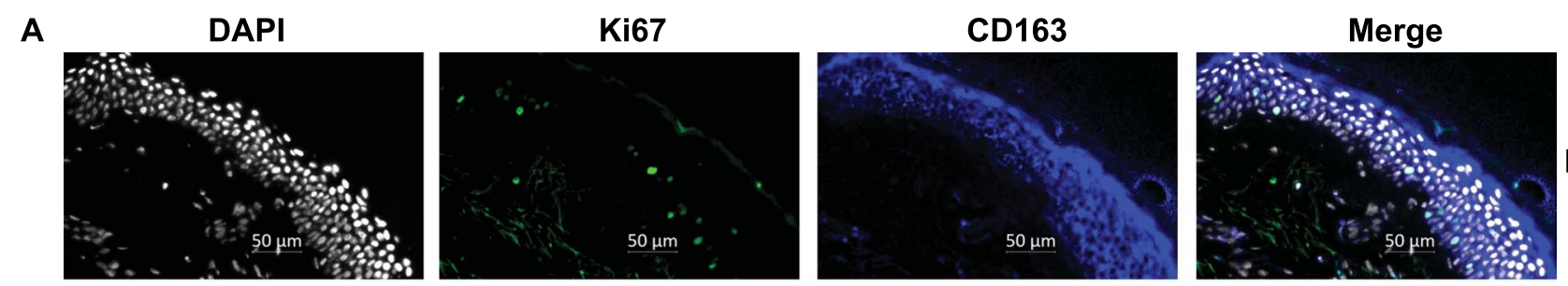

Normal
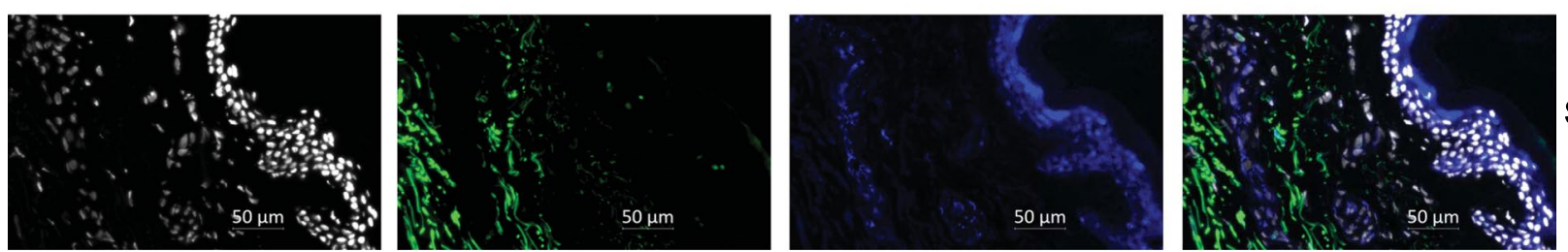

Saline

B

CD163+Ki67+
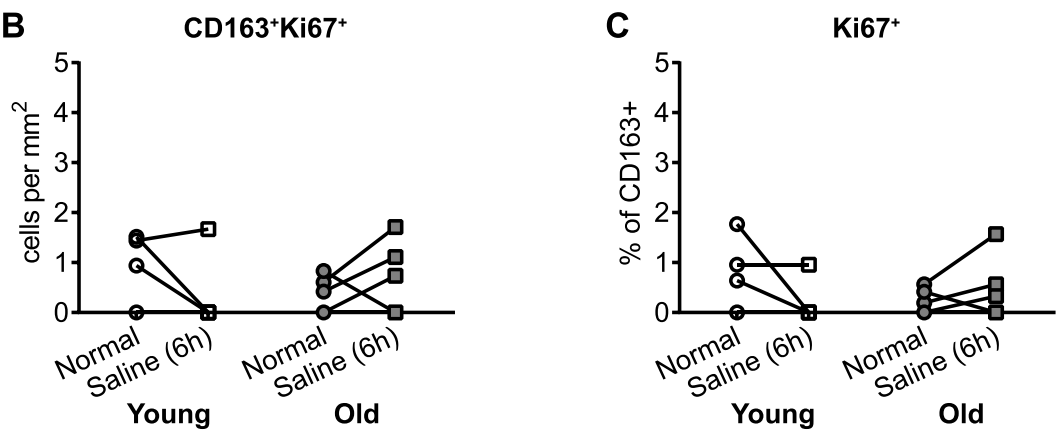

Extended Data Fig. 6 | Minimal Ki67 + macrophages are observed in saline-injected skin. A, representative staining of normal and saline-injected skin stained for DAPI (white), Ki67 (green), and CD163 (blue) and B, cumulative data showing the number of CD163 + Ki67+ and C, frequency of Ki67 + CD163 + cells in young (white; $n=4$ ) and old (grey $n=5$ ). Data assessed by paired t-test and no significance was found. 
A

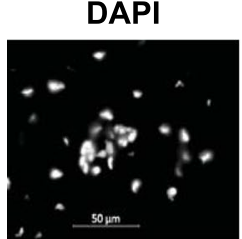

C

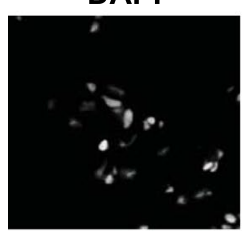

D
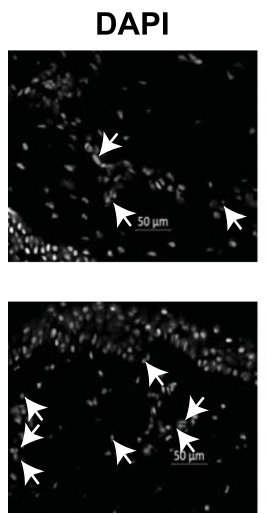

CCL2

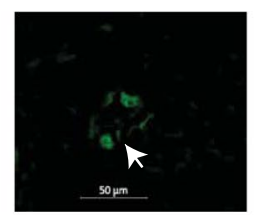

CCL2

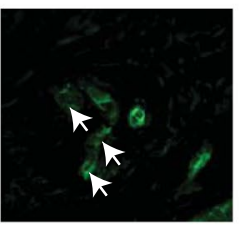

CCL2
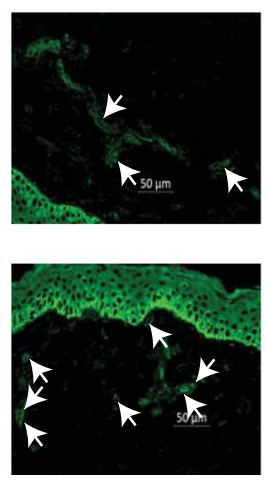

CD14

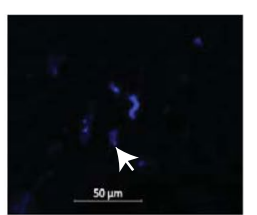

FSP1

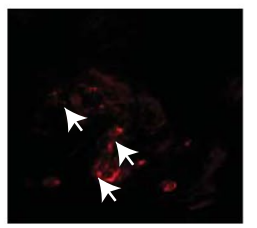

p16
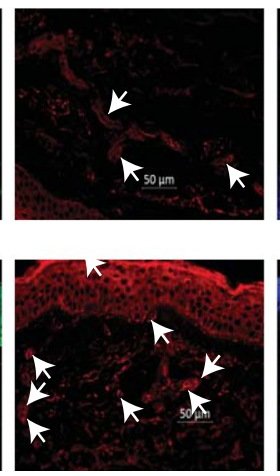

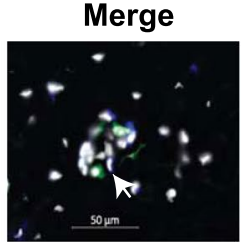

Merge

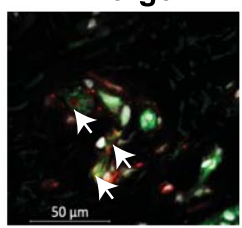

FSP1
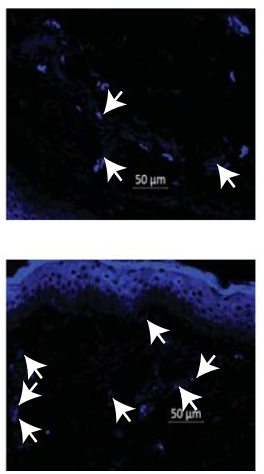

B

\section{$\mathrm{CD}{ }^{+} \mathrm{CCL2}^{+}$}

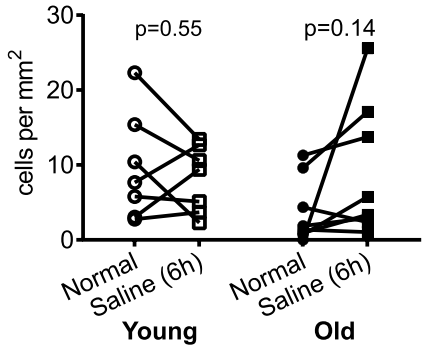

Extended Data Fig. 7 | Representative staining of monocyte chemoattractant expression after saline injection in the skin. A, representative images of saline-injected old skin stained with DAPI (white), CCL2 (green) and CD14 (blue) and B, cumulative data showing the number of CD14 + CCL2 + cells in normal and saline injected skin of young (white $n=7$ biologically independent individuals) and old skin (black $n=8$ biologically independent individuals). C, representative staining of saline-injected old skin of DAPI (white) CCL2 (green) and FSP1 (red) and D, representative images of normal and saline-injected old skin stained with DAPI (white), CCL2 (green), p16 (red) and FSP1 (blue). Arrows indicate double positive. E, cumulative data showing the frequency of CCL2 +FSP-1+ that express p16+ in old normal and saline-injected skin ( $n=7$ biologically independent individuals). $B$, and $E$, assessed with a paired t-test and no significance was found. 
DAPI

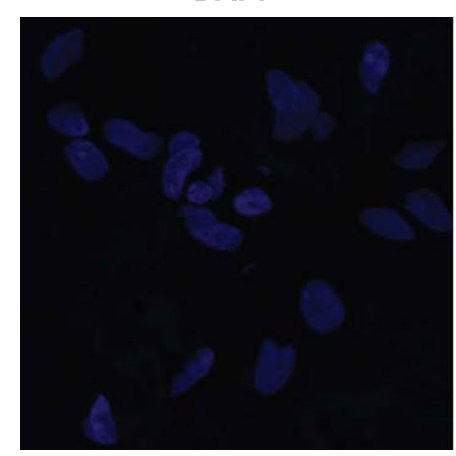

$\gamma \mathrm{H}_{2} \mathbf{A X}$

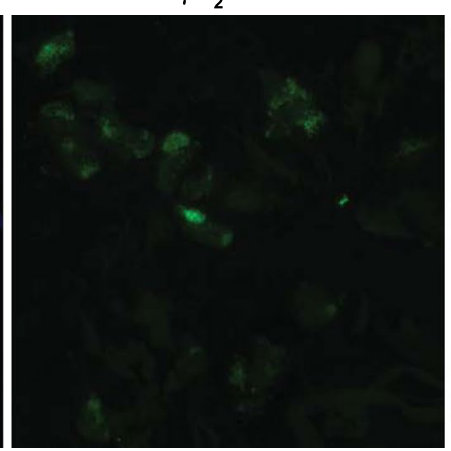

TAF and FSP-1

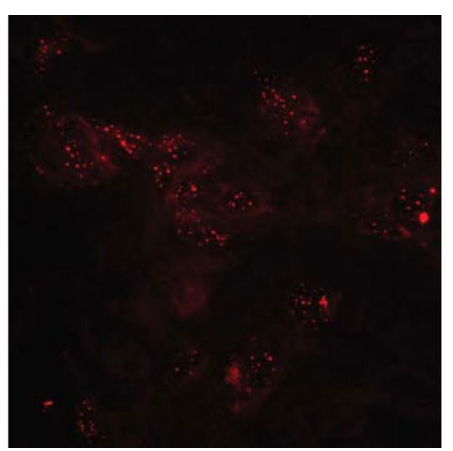

Merge

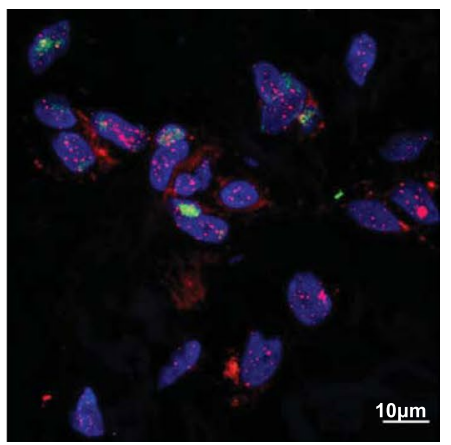

Extended Data Fig. 8 | Representative TAF staining Representative images of saline-injected old skin stained with DAPI (white), Telomere probe (intranuclear; red), FSP-1 (cell surface; red), and yH2AX (green). 

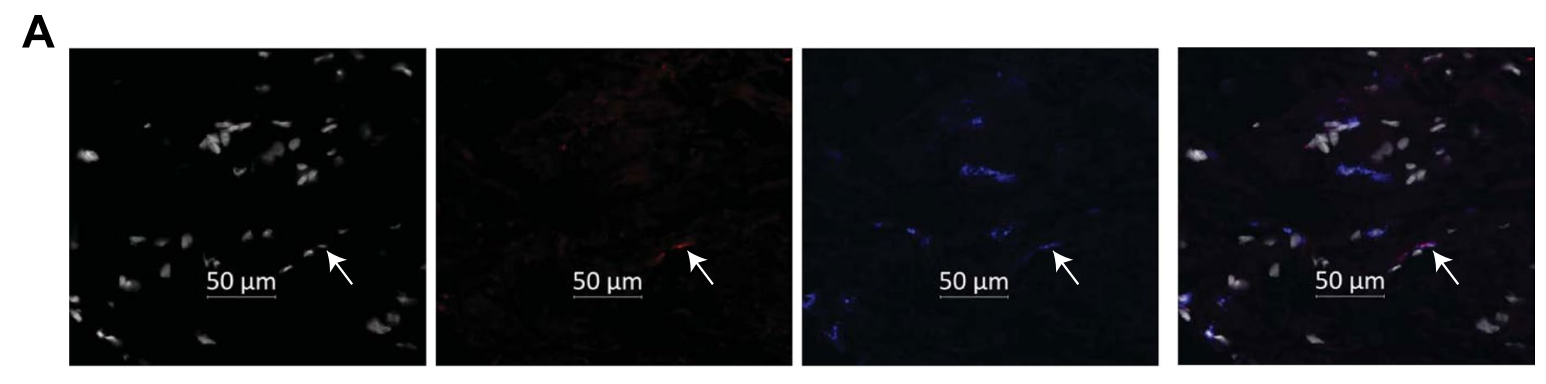

Normal
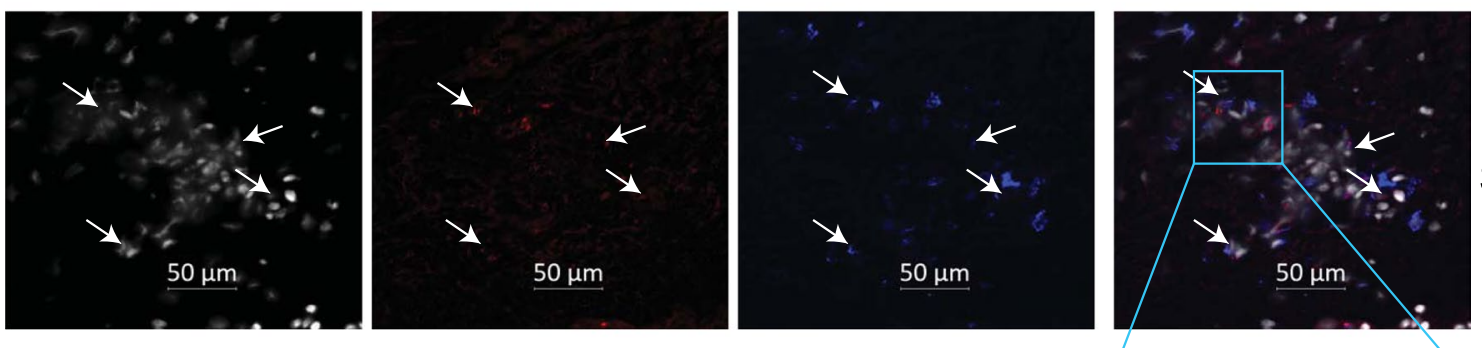

Old

B

\section{CD14-CoX2 ${ }^{+}$}
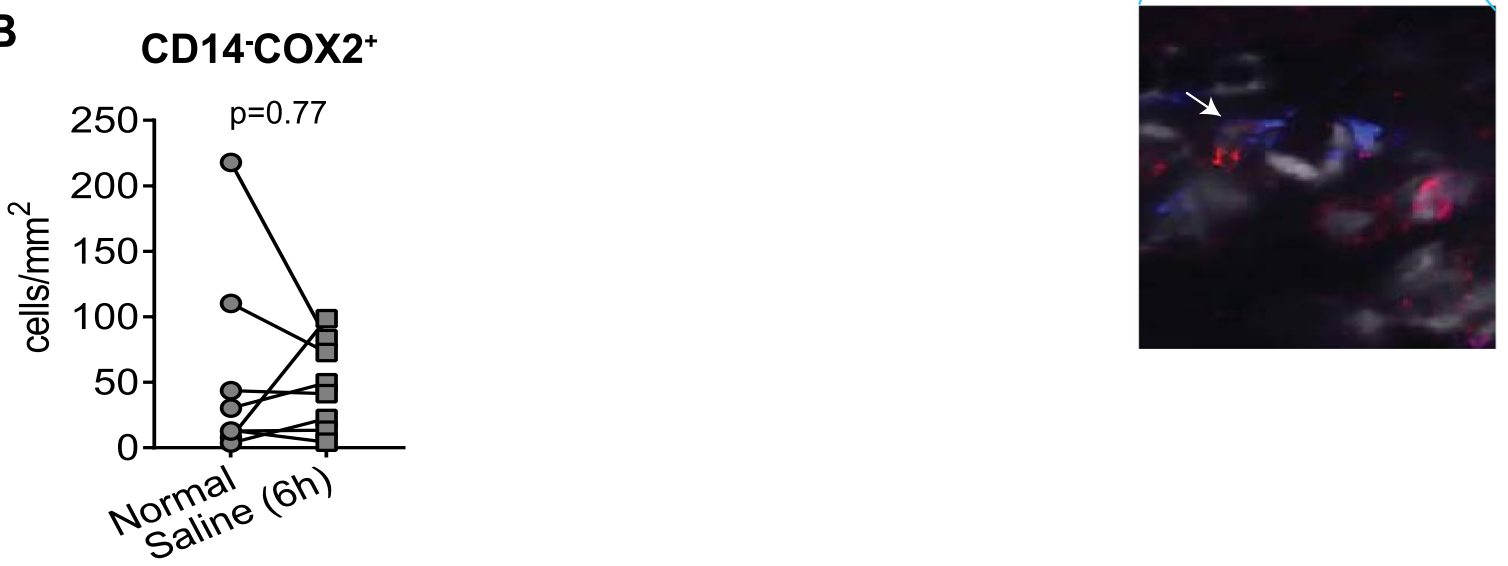

\section{Saline}

Extended Data Fig. 9 | CD14 and COX2 staining in normal and saline-injected older skin. A, Representative images of old normal and saline-injected skin sections stained with DAPI (white), COX (red) and CD14 (blue). B, cumulative data showing the number of CD14-COX2 + cells in normal and saline-injected old skin ( $n=8$ biologically independent individuals). 
A

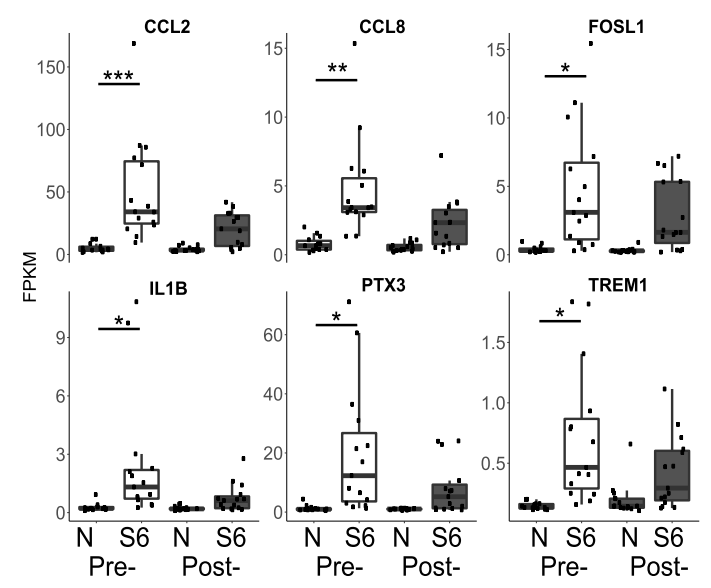

E

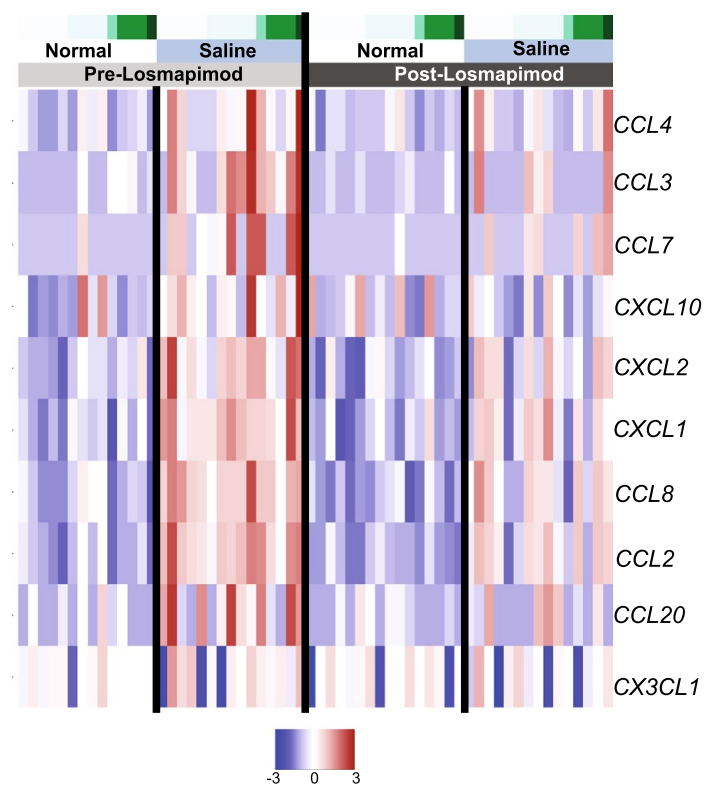

B

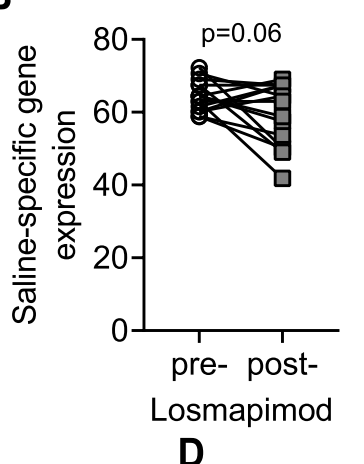

C

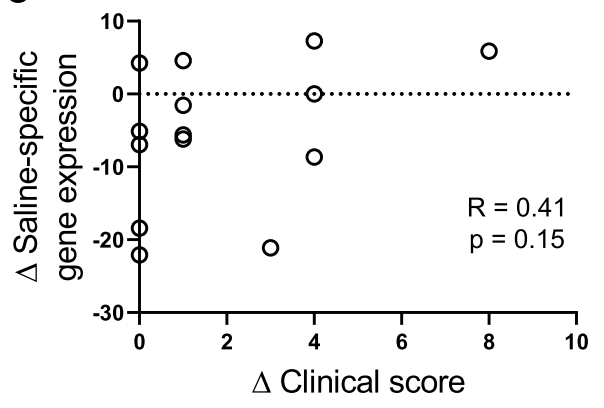

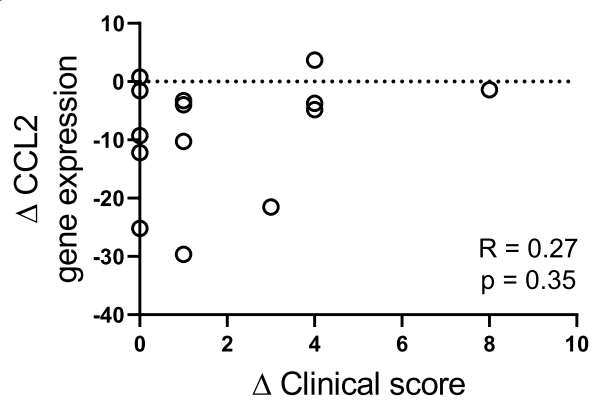

F

CCL2

$p=0.06$

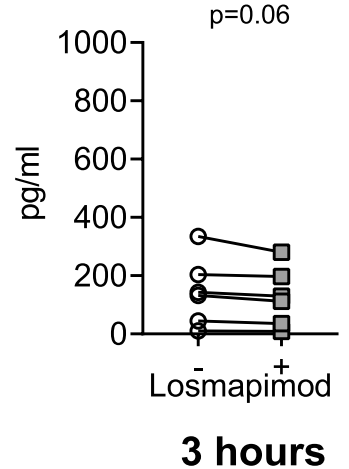

CCL2

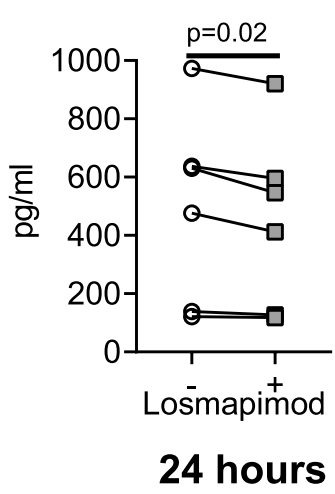

Extended Data Fig. 10 | Losmapimod reduces the expression of monocyte chemokines. A, top six genes upregulated in saline injected as compared to normal skin, when ranking by average fold change for genes significantly upregulated (adj.p <0.05) before and after Losmapimod pre-treatment (box shown as median, \pm interquartile range and error bars of $1.5^{\star} \mathrm{IQR}$ ). B, Saline-specific gene expression pre and post-Losmapimod and $C$, correlation between change in saline-specific gene expression or D, change in CCL2 gene expression and change in clinical score and pre- and post-Losampimod. E, heat map of gene expression of monocyte chemokines in normal and saline injected skin pre- and post-Losmapimod. F, Senescent fibroblasts were cultured with Losmapimod $(3 \mu \mathrm{M})$ for 3 or 24 hours, and supernatants were collected. CCL2 concentrations were assessed by cytometric bead array $(n=6)$. For $A-D n=15$ biologically independent individuals. $B$ and $F$ assessed by paired $t$ test and $C$ and $D$ assessed by Spearman's rank correlation test. ${ }^{\star}=p<0.05^{\star \star}=p<0.01^{\star \star \star}=p<0.001$. 


\section{Reporting Summary}

Nature Research wishes to improve the reproducibility of the work that we publish. This form provides structure for consistency and transparency in reporting. For further information on Nature Research policies, see our Editorial Policies and the Editorial Policy Checklist.

\section{Statistics}

For all statistical analyses, confirm that the following items are present in the figure legend, table legend, main text, or Methods section.

$\mathrm{n} / \mathrm{a} \mid$ Confirmed

X The exact sample size $(n)$ for each experimental group/condition, given as a discrete number and unit of measurement

Х $\square$ A statement on whether measurements were taken from distinct samples or whether the same sample was measured repeatedly

The statistical test(s) used AND whether they are one- or two-sided

Only common tests should be described solely by name; describe more complex techniques in the Methods section.

A description of all covariates tested

A description of any assumptions or corrections, such as tests of normality and adjustment for multiple comparisons

A full description of the statistical parameters including central tendency (e.g. means) or other basic estimates (e.g. regression coefficient)

AND variation (e.g. standard deviation) or associated estimates of uncertainty (e.g. confidence intervals)

For null hypothesis testing, the test statistic (e.g. $F, t, r$ ) with confidence intervals, effect sizes, degrees of freedom and $P$ value noted Give $P$ values as exact values whenever suitable.

For Bayesian analysis, information on the choice of priors and Markov chain Monte Carlo settings

For hierarchical and complex designs, identification of the appropriate level for tests and full reporting of outcomes

Estimates of effect sizes (e.g. Cohen's d, Pearson's $r$ ), indicating how they were calculated

Our web collection on statistics for biologists contains articles on many of the points above.

\section{Software and code}

Policy information about availability of computer code

Data collection no software was used

Data analysis Data was analysed in Graphpad Prism Version 8. Flow cytometry data was analysed in FlowJo Version X. Scripts used in the RNA-seq analysis can be found on the GitHub repository at https://github.com/barbarashih/arise_losmapimod

For manuscripts utilizing custom algorithms or software that are central to the research but not yet described in published literature, software must be made available to editors and reviewers. We strongly encourage code deposition in a community repository (e.g. GitHub). See the Nature Research guidelines for submitting code \& software for further information.

\section{Data}

Policy information about availability of data

All manuscripts must include a data availability statement. This statement should provide the following information, where applicable:

- Accession codes, unique identifiers, or web links for publicly available datasets

- A list of figures that have associated raw data

- A description of any restrictions on data availability

Data availability statement: RNAseq data that support the findings of this work, can be found RNAseq data that support the findings of this study have been deposited in NCBI Gene Expression Omnibus, accession code GSE130633. The rest of the data that support the findings of this study are available as source data. 
Please select the one below that is the best fit for your research. If you are not sure, read the appropriate sections before making your selection.

\ Life sciences

Behavioural \& social sciences

Ecological, evolutionary \& environmental sciences

For a reference copy of the document with all sections, see nature.com/documents/nr-reporting-summary-flat.pdf

\section{Life sciences study design}

All studies must disclose on these points even when the disclosure is negative.

$\begin{array}{ll}\text { Sample size } & \begin{array}{l}\text { The sample size required and the power calculations have been based on this data from our preliminary studies; The expected effect has been } \\ \text { determined on the basis of previous study performed by our group. For the Losmpamod study sample calculations we assumed an alpha level } \\ \text { of } 0.05 \text { (2-sided). A power of } 0.90 \text { was considered desirable. }\end{array}\end{array}$

Data exclusions Older people with VZV clinical scores over 3 were not recruited to take part in the study, this was a predetermined exclusion criteria based upon our previously published data (Vukmanovic-Stejic et al 2018; Vukmanovic-Stejic et al 2015; Agius et al 2009)

Replication $\quad$ All attempts at replication were successful. Each figure contains data from different individuals therefore each data point is an independent human replicate. See the figure legends for number of individuals used in each figure.

Randomization We did not randomise our study - the design of the study meant there was only one arm which meant all participants were treated the same.

Blinding Our study was not blinded as it was a clinical study rather then clinical trial. However all immunofluoresence counting was performed blinded to the assessor to prevent bias.

\section{Reporting for specific materials, systems and methods}

We require information from authors about some types of materials, experimental systems and methods used in many studies. Here, indicate whether each material, system or method listed is relevant to your study. If you are not sure if a list item applies to your research, read the appropriate section before selecting a response.

\begin{tabular}{l|l} 
Materials \& experimental systems \\
\hline n/a & Involved in the study \\
$\square$ & $\bigotimes$ Antibodies \\
$\triangle$ & $\square$ Eukaryotic cell lines \\
$\square$ & $\square$ Palaeontology and archaeology \\
$\square$ & $\square$ Animals and other organisms \\
$\searrow$ & $\square$ Clinical data \\
$\square$ & $\square$ Dual use research of concern
\end{tabular}

\begin{tabular}{l} 
Methods \\
\hline n/a Involved in the study \\
$\square$ \\
$\square$ ChIP-seq \\
$\square$ Flow cytometry \\
$\square$ MRI-based neuroimaging
\end{tabular}

Antibodies

Antibodies used

For full list of antibodies and dilutions used please see Supplementary Tables 2 and 3.

Validation

Antibodies were validated by the manufacturer and where there is an exception to this (TelC probe and Ki67-FITC) it is detailed in Supplementary Table 2 how they were validated. Optimum antibody concentration was calculated by titrating the antibody prior to staining the samples - see Supplementary Tables 2 and 3.

\section{Human research participants}

Policy information about studies involving human research participants

Population characteristics

Recruitment

Ethics oversight
See Supplementary Table 1 which lists population characteristics. For the study involving Losmapimod, we recruited 42 healthy older adults ( $>65$ years); 19 males and 23 females with a mean age of 70.7 years old ( $95 \% \mathrm{Cl} 69-72.3$ years old). Only Caucasian European individuals were included in the study

Older Participants were recruited from either in person or email based communication to older groups/centres in London by research nurses and research scientists. For the younger participants, they were recruited from the website 'Call for Participants'. We don't believe there was a recruitment bias.

Ethics Committee Queen's Square (London) and institutional review board (UCL R\&D). 
Plots

Confirm that:

Х The axis labels state the marker and fluorochrome used (e.g. CD4-FITC).

Х The axis scales are clearly visible. Include numbers along axes only for bottom left plot of group (a 'group' is an analysis of identical markers).

$\bigotimes$ All plots are contour plots with outliers or pseudocolor plots.

\A numerical value for number of cells or percentage (with statistics) is provided.

\section{Methodology}

Sample preparation

Instrument

Software

Cell population abundance

Gating strategy
Skin biopsies $(5 \mathrm{~mm}$ ) were taken from normal and saline injected skin ( 6 or 24-hours post-injection) and disaggregated by overnight incubation $(370 \mathrm{C}$ ) in either $0.8 \mathrm{mg} / \mathrm{ml}$ collagenase IV (Sigma Aldrich) with 20\% FCS or a whole skin digestion kit from Miltenyi Biotec (Cambridge, U.K.). Single cell suspensions were obtained and subsequently stained

BD Fortessa using FACSDIVA software

FlowJo version $\mathrm{X}$ was used to analyse the samples.

Samples were not sorted, whole skin was assessed by flow cytometry.

CD45+ selected, then single cell gate (SSCH vs SSC A) and then another singlet gate used (FSCH vs FSCA). Cells were identified as mononuclear phagocytes if they were Lineage negative for CD3, CD19, CD20 CD56 and HLA-DR+. Gating was based upon FMO controls performed in matched blood donors.

$\bigotimes$ Tick this box to confirm that a figure exemplifying the gating strategy is provided in the Supplementary Information. 